\title{
Transport of Plasma Free Fatty Acids and Triglycerides in Man: a Theoretical Analysis
}

\author{
David M. Shames, Arthur Frank, Daniel Steinberg, and \\ MONES BERMAN \\ From the Mathematical Research Branch, National Institute of Arthritis and \\ Metabolic Diseases and the Laboratory of Molecular Diseases, National Heart \\ Institute, National Institutes of Health, Bethesda, Maryland 20014, and the \\ Division of Metabolic Disease, Department of Medicine, University of \\ California at San Diego, La Jolla, California 92037
}

A в S T R A C T Three different multicompartmental models of free fatty acid (FFA) and very low density lipoprotein triglyceride fatty acid (VLDL-TGFA) transport in man are formulated from plasma FFA and VLDL-TGFA tracee and tracer data collected over a $24 \mathrm{hr}$ interval after the injection of palmitate $-{ }^{14} \mathrm{C}$. All modeling and data fitting were performed on a digital computer using the SAAM program. Structural differences in the three models relate to the position of the slowly turning over compartment required to generate the late portion of the plasma VLDL-TGFA tracer data. The positions of this slow compartment are along the hepatic pathway from FFA to VLDL-TGFA (model A) or in the distribution system of VLDL-TGFA (model $B$ ) or in the distribution system of FFA (model C). Although all three models are equally consistent with our experimental data and are supported by observations of others, each reveals inconsistency with some data obtained from the literature. Consequently, a combination model of FFA-TGFA transport, incorporating properties of models $\mathrm{A}, \mathrm{B}$, and $\mathrm{C}$ would be more consistent with all available data. Experiments that would help to determine the quantitative significance of each of the slow compartments in the combination model are suggested.

Several other models suggesting recycling of plasma VLDL-TGFA through the plasma FFA pool, kinetic

Dr. Shames' present address is the Department of Radiology, University of California Medical Center, San Francisco, Calif. 94122.

Dr. Frank's present address is the Office of Economic Opportunity, Washington, D. C.

Dr. Steinberg's present address is the Division of Metabolic Disease, Department of Medicine, University of California at San Diego, La Jolla, Calif. 92037.

Received for publication 20 January 1970 and in revised form 15 June 1970. heterogeneity of the plasma VLDL-TGFA pool, and contamination of plasma VLDL-TGFA radioactivity with low density lipoprotein (LDL) TGFA radioactivity were tested. The first model does not explain the late portion of the plasma VLDL-TGFA tracer data. The second and third models, while consistent with our tracee and tracer data, have steady-state implications with respect to the extent of kinetic heterogeneity and size of the LDL-TGFA contaminant that make them unlikely.

Assumptions underlying other investigators' models of FFA and TGFA transport in man are reviewed within the logical framework of our models. Quantitative differences among the various models are shown by evaluating all of the models with respect to a common set of plasma FFA and VLDL-TGFA data.

\section{INTRODUCTION}

During the last $10 \mathrm{yr}$ several quantitative models have been proposed to describe plasma free fatty acid (FFA) and triglyceride fatty acid (TGFA) transport in man. Most of these models have been based on the responses of labeled lipid moieties in the plasma following the injection or infusion of radioactive glycerol, FFA or TGFA. Farquhar, Gross, Wagner, and Reaven (1) and Nestel (2) injected glycerol- ${ }^{3} \mathrm{H}$ and $\mathrm{FFA}-{ }^{14} \mathrm{C}$, respectively, and estimated the production rate of TGFA in very low density lipoprotein (VLDL) from measurements of plasma VLDL-TGFA specific activity (SA). A somewhat different approach was used by Friedberg, Klein, Trout, Bogdonoff, and Estes (3) to estimate the contribution of plasma FFA to plasma TGFA following the injection of FFA- ${ }^{14} \mathrm{C}$. Ryan and Schwartz (4) proposed a method, also used by Sailer, Sandhofer, and Braunsteiner (5) and Miller, Bartz, and Durham (6), 


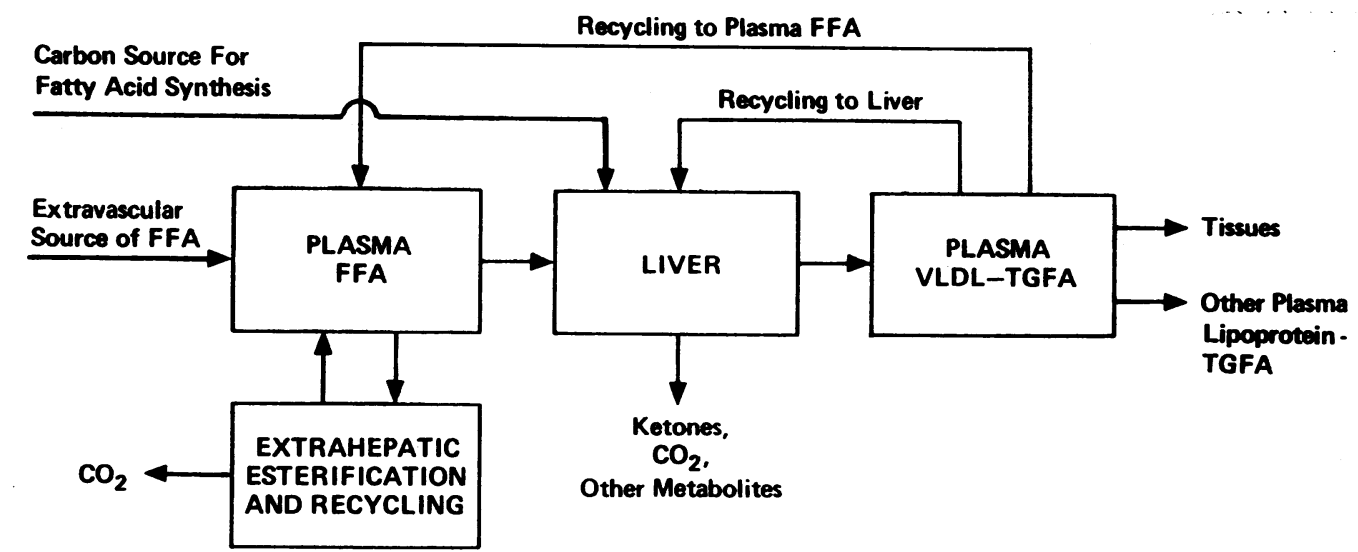

FIGURE 1 Block diagram describing some of the main pathways of FFA and VLDL-TGFA transport.

to estimate the extent of FFA incorporation into TGFA. It was based on plasma FFA and TGFA SA data obtained during a continuous infusion of FFA $-{ }^{14} \mathrm{C}$. Havel (7) modified this method (Havel model II) and also obtained SA data on FFA and VLDL-TGFA in hepatic vein blood to allow a better estimate of the production rate of VLDL-TGFA (Havel model I). A multicompartmental model based on SA of plasma FFA and total TGFA collected over a $4 \mathrm{hr}$ interval was formulated by Eaton, Berman, and Steinberg (8) to estimate several measures of FFA and TGFA transport. Some of these approaches to FFA-TGFA kinetics were recently reviewed by Baker (9).

Quarfordt et al. (10) have continued the investigations of Eaton et al. (8) in normal and hypertriglyceridemic subjects on normal and high carbohydrate diets. They measured specifically the SA of TGFA in plasma VLDL and extended their observations over a $24 \mathrm{hr}$ interval following the injection of palmitate- ${ }^{14} \mathrm{C}$. To deal with these latest studies and others in the literature, the model of Eaton et al. (8) is developed further in this report. Several different compartmental models are formulated that are equally consistent with the experimental data but have different physiological implications. These implications are carefully analyzed in an attempt to determine the applicability of each model to lipid transport in man. The results of this analysis suggest additional experiments that might clarify those measures of lipid transport which remain quantitatively uncertain. An attempt is made to explain some of the wide variation in estimates of measures of lipid transport reported by different groups of investigators.

\section{Development of Compartmental Model}

A. Physiological considerations. A schematic inputoutput diagram of the basic metabolic pathways considered in construction of the model are shown in Fig. 1.
Plasma FFA are mainly derived from extravascular sources (primarily adipose tissue), although some may come directly from intravascular hydrolysis of esterified fatty acids contained in plasma lipoproteins. Pathways taken by FFA leaving the plasma include hepatic and extrahepatic esterification, exchange with extravascular pools (extracellular and intracellular), and tissue oxidation to $\mathrm{CO}_{2}$. A fraction of the plasma FFA extracted by the liver and of the fatty acid synthesized de novo in the liver is oxidized to $\mathrm{CO}_{2}$ or ketone bodies, and some is subsequently secreted into the plasma as VLDL-TGFA. Pathways for plasma VLDL-TGFA include uptake by peripheral tissues, conversion to other plasma lipoproteins, and recycling to liver or to plasma FFA.

$B$. Kinetic considerations: qualitative. To be acceptable a model must be compatible with all of the kinetic data available from many sources. These include data on plasma $\mathrm{FFA}-{ }^{14} \mathrm{C}$, plasma VLDL-TGFA- ${ }^{14} \mathrm{C}$, and ${ }^{14} \mathrm{CO}_{2}$ expiration after the injection or infusion of palmitate $-{ }^{14} \mathrm{C}$. Other important constraints are imposed by the requirement that steady-state calculations for pool sizes of plasma FFA and VLDL-TGFA and hepatic TGFA and for the rate of total $\mathrm{CO}_{2}$ excretion must be compatible with observed or estimated values. Taking all of these factors into account, three models were formulated and tested against experimental data (models A, B, and C in Fig. 1 of the companion paper). ${ }^{1}$ Symbols used to describe model parameters and various composite measures of these parameters are defined in Table I.

The basis for the compartmental structure of the three models can be readily understood in qualitative terms by careful attention to the shapes of plasma FFA

\footnotetext{
${ }^{1}$ All three models are equally consistent with our plasma FFA and VLDL-TGFA tracer and tracee data, yet each has distinct physiological implications relating to the position of the slowly turning over compartment (dashed circles).
} 
TABLE I

Definitions of Symbols*

\begin{tabular}{|c|c|}
\hline \multicolumn{2}{|c|}{ Symbols relating to models $\mathrm{A}, \mathrm{B}$, and C } \\
\hline$\lambda_{i j}$ & $\begin{array}{l}\text { Fractional rate of transport of activity or mass } \\
\text { to compartment } i \text { from compartment } j\left(\min ^{-1}\right)\end{array}$ \\
\hline$\lambda_{0 j}$ & $\begin{array}{l}\text { Fractional rate of irreversible transport of } \\
\text { activity or mass out of system from compart } \\
\text { ment } \mathrm{j}\left(\min ^{-1}\right)\end{array}$ \\
\hline$\lambda_{\mathrm{jj}}$ & $\begin{array}{l}\text { Fractional rate of irreversible and reversible } \\
\text { transport of activity or mass out of compart } \\
\text { ment } \mathrm{j}\left(\mathrm{min}^{-1}\right)\end{array}$ \\
\hline$\rho_{\mathrm{ij}}$ & 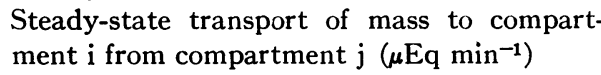 \\
\hline$\rho_{0 \mathrm{j}}$ & 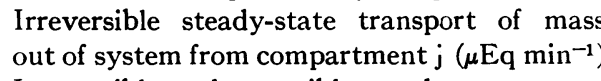 \\
\hline$\rho_{\mathrm{jj}}$ & $\begin{array}{l}\text { Irreversible and reversible steady-state trans- } \\
\text { port of mass out of compartment } j\left(\mu \mathrm{Eq} \min ^{-1}\right)\end{array}$ \\
\hline $\mathbf{M}_{\mathbf{j}}$ & Steady-state mass of compartment $\mathrm{j}(\mu \mathrm{Eq})$ \\
\hline $\mathrm{U}_{\mathrm{j}}$ & $\begin{array}{l}\text { Steady-state transport of mass into compart } \\
\text { ment } \mathrm{j} \text { from outside of system }\left(\mu \mathrm{Eq} \min ^{-1}\right)\end{array}$ \\
\hline$\alpha$ & $\begin{array}{l}\text { Fraction of total plasma FFA incorporated } \\
\text { into plasma VLDL-TGFA by way of the fast } \\
\text { pathway for FFA } \rightarrow \text { VLDL-TGFA }\end{array}$ \\
\hline \multicolumn{2}{|c|}{ Symbols relating to FFA and TGFA transport in general } \\
\hline $\mathrm{M}_{\text {TGFA }}$ & Plasma VLDL-TGFA mass $(\mu \mathrm{Eq})$ \\
\hline$\lambda_{\mathrm{TG}}$ & $\begin{array}{l}\text { Fractional rate of turnover of plasma VLDI } \\
\text { TGFA }\left(\mathrm{min}^{-1}\right)\end{array}$ \\
\hline$\rho$ TGFA & $\begin{array}{l}\text { Steady-state rate of production of VLDL } \\
\text { TGFA }\left(\mu \mathrm{Eq} \min ^{-1}\right)\end{array}$ \\
\hline$\rho$ FFA $\rightarrow$ TGFA & $\begin{array}{l}\text { Steady-state rate of incorporation of plasma } \\
\text { FFA into plasma VLDL-TGFA }\left(\mu \mathrm{Eq} \mathrm{min}^{-1}\right)\end{array}$ \\
\hline $\mathrm{U}_{\mathrm{FFA}}$ & $\begin{array}{l}\text { Steady-state rate of delivery of FFA (non } \\
\text { recycling) into plasma }\left(\mu \mathrm{Eq} \mathrm{min}^{-1}\right)\end{array}$ \\
\hline $\mathrm{FC}$ & $\begin{array}{l}\text { Fraction of FFA activity extracted by liver } \\
\text { which is secreted as VLDL-TGFA activity }\end{array}$ \\
\hline
\end{tabular}

* Terminology used throughout is that recommended by the Task Group on Tracer Kinetics of the International Commission on Radiological Units and Measurements (29).

and VLDL-TGFA SA curves obtained after the injection of palmitate ${ }^{14} \mathrm{C}$. A typical set of such data from a normal subject (study $1 \mathrm{~A}$ in companion paper) is presented in Fig. 2. The biexponential function required to fit the FFA tracer data over 90 min implies exchange of plasma FFA with at least one other compartment (compartment 2 in models $\mathrm{A}, \mathrm{B}$, and C) (10). The VLDL-TGFA tracer data exhibit an initial time delay before any significant activity is observed in the plasma VLDL-TGFA pool. This delay has been previously noted by Baker and Schotz (11) in the rat and by Eaton et al. (8) in man. The VLDL-TGFA tracer data also exhibit a flattening at the top of the curve as well as a further delay in the time $\left(t_{\max }\right)$ at which maximum activity is reached. The $t_{\max }$ value in some patients with hypertriglyceridemia can occur as late as $240 \mathrm{~min}$ (10).
Both of these features of the VLDL-TGFA tracer curve (flattening at top and delayed $t_{\max }$ ) suggest that plasma FFA enroute to VLDL-TGFA mix in an intermediate hepatic pool turning over more slowly than that producing the initial delay before VLDL-TGFA activity appears in the plasma. This intermediate hepatic pool is introduced in all three models as compartment 4 (Fig. 1 of the companion paper). ${ }^{1}$ After $t_{\max }$ the VLDL-TGFA plasma activity initially disappears in what appears to be a first-order fashion. However, a slow component clearly emerges beyond $500 \mathrm{~min}$. This slow component has been found by all investigators who have carried out observations over a long enough time interval $(1,2,10$, 12). Extensive testing of various models showed that the slow component can be generated by a slowly turning over compartment (hashed circles of Fig. 1 of the companion paper) positioned either in the FFA distribution system (model $\mathrm{C}$ ), along the hepatic pathway from FFA to VLDL-TGFA (model $\mathrm{A}$ ), or in the distribution system of VLDL-TGFA (model B). Each one of these three positions has distinct physiological implications and forms the basis for the differences among models $\mathrm{A}, \mathrm{B}$, and $\mathrm{C}$.

C. Kinetic considerations: quantitative. Using the SAAM digital computer program $(13,14)$, an attempt was made to fit model $\mathrm{A}$ to the tracer and tracee data shown in Fig. 2. It was found that the model was nonunique, i.e., certain parameters could assume a wide range of values and still fit the data equally well (15). To obtain uniqueness the following statistical constraints were imposed on the parameters of the model: (a) $\lambda_{5,4}=0.0150 \mathrm{~min}^{-1} \pm 20 \%$, (b) $\lambda_{1,2}=0.0175 \mathrm{~min}^{-1}$ $\pm 20 \%$.

The value of $\lambda_{5,4}$ in constraint 1 was chosen as a population mean after trying various values ranging from $0.005 \mathrm{~min}^{-1}$ to $0.05 \mathrm{~min}^{-1}$. Values of $\lambda_{5,4}$ between $0.01 \mathrm{~min}^{-1}$ and $0.02 \mathrm{~min}^{-1}$ generated a degree of flattening at the top of the VLDL-TGFA tracer curve and delay in $t_{\max }$ most consistent with data from all the normal and hyperglyceridemic studies in the present series (10). Effects of changes in $\lambda_{5,4}$ on the values of other parameters in the model are discussed under Model Testing.

The second constraint was required because of the inability to determine uniquely the slope of the second exponential component of the plasma FFA tracer data. A value for $\lambda_{1,2}$ was therefore chosen to be consistent (within the statistical limits defined) with the plasma FFA tracer data obtained by Eaton et al. (8). It is shown later (see Model Testing) that most measures of lipid transport with respect to models $\mathrm{A}, \mathrm{B}$, and $\mathrm{C}$ are, in any case, relatively insensitive to the actual magnitude of this slope. 


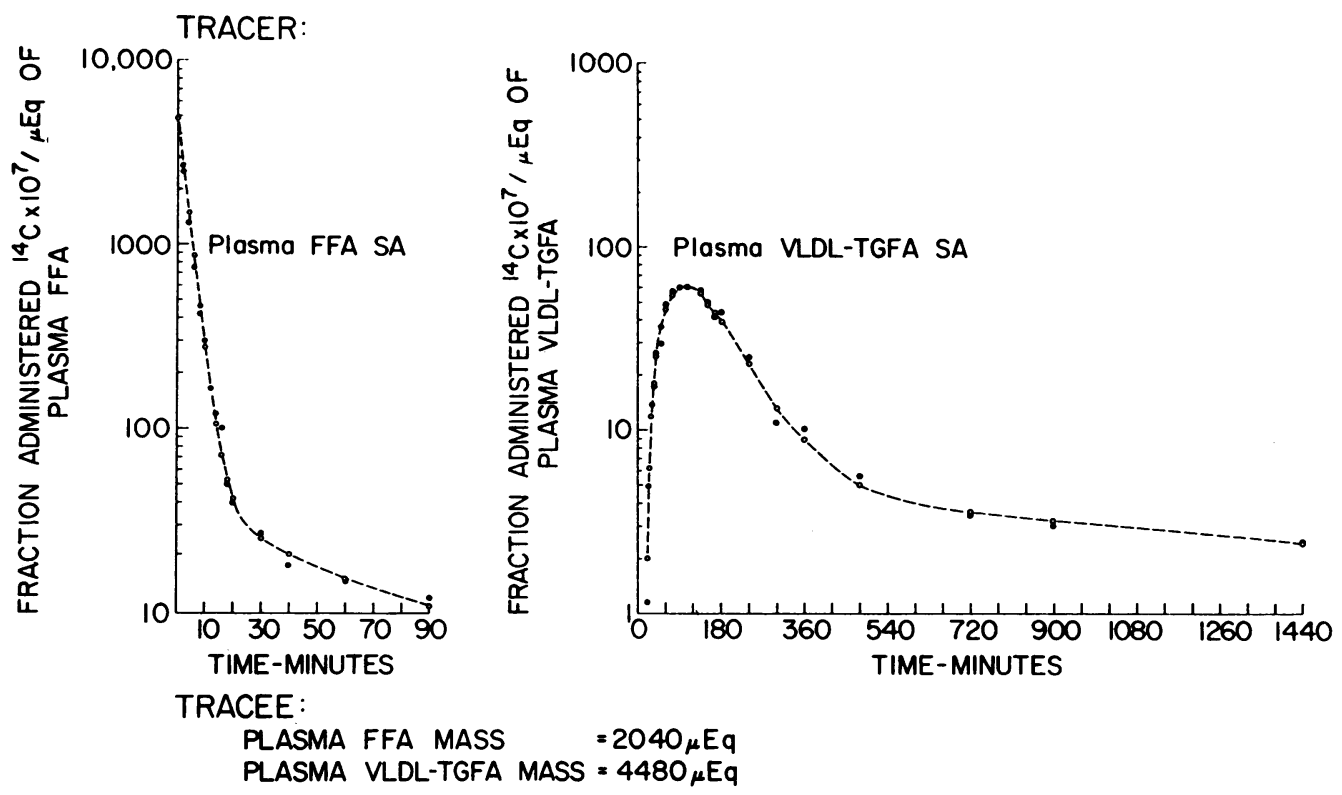

Figure 2 Typical plasma FFA and VLDL-TGFA tracer and tracee data obtained from a normal subject following the injection of palmitate $-{ }^{14} \mathrm{C}$. The fit of computer-generated data from model A to the experimental data is also shown. Open circles represent calculations; closed circles represent observations.

In addition to these statistical constraints, $\lambda_{0,1}$ was assigned a lower limit based on estimates of the average normal fractional rate of plasma FFA oxidation to CO. Assuming an average of 17 carbon atoms per molecule of FFA and a $\mathrm{CO}_{2}$ excretion rate of $10 \mathrm{mIM}$ $\min ^{-1}$ of which $25 \%$ is derived from plasma FFA through relatively fast oxidative pathways (16), the steady-state rate of FFA oxidation to $\mathrm{CO}_{2}$ is approximately $150 \mu \mathrm{Eq} \mathrm{min}^{-1}$. The minimal limit for $\lambda_{0,1}$ was therefore set equal to $150 \mu \mathrm{Eq} \mathrm{min}^{-1} / \mathrm{M}_{1}$. In the particular study shown in Fig. 2, the plasma FFA mass $\left(M_{1}\right)$ was $2040 \mu \mathrm{Eq}$, and consequently the minimal limit allowed for $\lambda_{0,1}$ in fitting model $\mathrm{A}$ to the data was 0.074 $\min ^{-1}$.

In obtaining steady-state solutions, the values of the plasma FFA and VLDL-TGFA pool sizes $\left(\mathrm{M}_{1}\right.$ and $\mathrm{M}_{5}$, respectively) were assumed to be known precisely and were calculated from measured plasma concentrations assuming a distribution space equal to plasma volume $(41.3 \mathrm{ml} / \mathrm{kg}$ of body weight). The tracer data of palmitate ${ }^{14} \mathrm{C}$ were assumed to be representative of all fatty acids. Since the steady-state rate of inflow of FFA into the plasma $\left(U_{1}\right)$ from outside the system was the only inflow allowed in models $\mathrm{A}$ and $\mathrm{B}$, knowledge of both $M_{1}$ and $M_{5}$ acted as a constraint on the tracer solution of these two models. To obtain correct calculations for $\mathrm{M}_{\mathrm{s}}$ in model $\mathrm{C}$ another source of fatty acids for VLDLTGFA synthesis $\left(U_{s}\right)$ was required in addition to that from plasma FFA. Because of the requirement for two steady-state inflows ( $U_{1}$ and $U_{5}$ ) instead of one, the tracer solution of model $\mathrm{C}$ was not rigidly constrained by the explicit values for $M_{1}$ and $M_{s}$, except that the sum of the $U$ values had to be compatible with the observed steady state.

The excellent fit of computer-generated data (using model A) to the experimental data of study $1 \mathrm{~A}$ is shown in Fig. 2. Models $B$ and $C$ were then evaluated using the theoretical data simulated from the calculated least squares fit of model $\mathrm{A}$ to the experimental data. This was done to avoid eliciting differences in parameter values among models $\mathrm{A}, \mathrm{B}$, and $\mathrm{C}$ that might be related only to random fluctuation of the experimental data. By analyzing the experimental data with respect to the three models in this way, differences in the parameter values shown below are due entirely to the intrinsic structural differences of the three models. Models $\mathrm{B}$ and $\mathrm{C}$ fit the theoretical data simulated from model $\mathrm{A}$ within about $1 \%$. In other words, with appropriate values for rate constants, the three models shown in Fig. 1 of the companion paper are all equally compatible with the experimental data. Parameter values, measures derived from them, and estimated uncertainties for models A, B, and C are shown in Table II. The estimates of uncertainties for models $\mathrm{B}$ and $\mathrm{C}$ are based on an assumed $5 \%$ random fluctuation in the theoretical data. Any choice among these models must be based, then, on data other than those shown in Fig. 2. The following sections analyze the similarities and differ- 
TABLE II

Intercomparison of Parameters and Steady-State Solutions Obtained on Appyling Models $A, B$, and C to a Common Data Set*

\begin{tabular}{|c|c|c|c|c|c|c|c|}
\hline $\begin{array}{l}\text { Parameter } \\
\text { or measure }\end{array}$ & Units & Model A & PSD $\ddagger$ & Model B & PSD\& & Model C & PSDS \\
\hline$\lambda_{31}$ & $\min ^{-1}$ & 0.0390 & 24 & 0.0209 & 24 & 0.0202 & 20 \\
\hline$\lambda_{21}$ & “" & 0.0369 & 17 & 0.0369 & 2 & 0.0199 & 4 \\
\hline$\lambda_{12}$ & “ & 0.0177 & 13 & 0.0179 & 2 & 0.0295 & 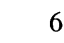 \\
\hline$\lambda_{8.1}$ & “" & - & - & - & - & 0.129 & 3 \\
\hline$\lambda_{1,8}$ & “ & 一 & - & - & - & 0.00103 & 6 \\
\hline$\lambda_{0,1}$ & “ & 0.223 & 8 & 0.241 & 10 & 0.130 & 2 \\
\hline$\lambda_{5,4}$ & “ & 0.0150 & 25 & 0.0150 & 21 & 0.0150 & 25 \\
\hline$\lambda_{5,6}$ & “ & 0.000546 & 31 & 一 & - & - & 一 \\
\hline$\lambda_{5,7}$ & “" & - & - & 0.00102 & 4 & - & 一 \\
\hline$\lambda_{0,5}$ & “" & 0.0178 & 25 & 0.00952 & 24 & 0.0161 & 20 \\
\hline$\lambda_{7,5}$ & “" & - & - & 0.00780 & 25 & - & - \\
\hline$\lambda_{5, j}$ & “" & 0.0178 & 25 & 0.0173 & 25 & 0.0161 & 20 \\
\hline$\alpha$ & & 0.52 & - & 1.0 & - & 1.0 & - \\
\hline$M_{1}$ & $\mu E_{q}$ & 2040 & - & 2040 & - & 2040 & - \\
\hline $\mathrm{M}_{5}$ & “" & 4480 & - & 4480 & - & 4480 & - \\
\hline $\mathrm{M}_{2}$ & “" & 4250 & 24 & 4250 & 2 & 1379 & 6 \\
\hline $\mathrm{M}_{4}$ & “ & 2749 & 34 & 2851 & 30 & 2749 & 20 \\
\hline$M_{6}$ & “ & 70339 & 35 & - & - & - & - \\
\hline $\mathrm{M}_{7}$ & “" & - & - & 35224 & 27 & - & - \\
\hline $\mathrm{M}_{8}$ & “ & - & - & - & - & 255890 & 6 \\
\hline $\mathrm{U}_{1}$ & $\mu E q$ min $^{-1}$ & 534 & 10 & 534 & 2 & 306 & 3 \\
\hline $\mathrm{U}_{5}$ & $“$ & - & - & - & - & 30.8 & 5 \\
\hline$\rho_{3,1}$ & $\mu E q \min ^{-1}$ & 79.6 & 27 & 42.6 & 24 & 41.2 & 20 \\
\hline$\rho_{0,5}$ & “ & 79.6 & 27 & 42.6 & 25 & 72.1 & 18 \\
\hline$\rho_{5,4}$ & “" & 41.2 & 25 & 42.6 & 27 & 41.2 & 20 \\
\hline$\rho_{5,6}$ & " & 38.4 & 30 & - & - & - & 一 \\
\hline$\rho_{5,7}$ & “ & - & 一 & 35 & 10 & - & - \\
\hline$\rho_{0,1}$ & “" & 455 & 8 & 492 & 10 & 265 & 12 \\
\hline$\rho_{2,1}$ & “" & 75.2 & 17 & 75.2 & 6 & 40.7 & 5 \\
\hline$\rho_{8,1}$ & “" & - & - & - & - & 263 & 4 \\
\hline$\rho_{5,5}$ & “" & 79.6 & 27 & 77.6 & 20 & 72.1 & 18 \\
\hline$\rho_{5,4} / \rho_{5,5}$ & & 0.52 & 26 & 0.55 & 23 & 0.57 & 18 \\
\hline
\end{tabular}

* Models $\mathrm{B}$ and $\mathrm{C}$ were fitted to theoretical data simulated from the least squares fit of of model $A$ to data of study $1 \mathrm{~A}$. The value for $\lambda_{5,4}$ was constrained $(0.015 \pm 20 \%)$ as discussed in the text.

$\ddagger$ Per cent SD.

\$ Uncertainties of models $\mathrm{B}$ and $\mathrm{C}$ are based on an assumed $5 \%$ random fluctuation in the theoretical data.

ences among the three models and the bases for choosing among them.

D. Comparison of parameters and steady-state solutions of models $A, B$, and $C$. Parameter differences having particular physiological significance in the three models (Table II) include $\lambda_{3,1}$ (fractional rate of hepatic uptake of plasma FFA enroute to plasma VLDLTGFA) and $\lambda_{0,5}$ (fractional rate of irreversible loss of plasma VLDL-TGFA activity). Since in models $B$ and $C$ there is no slowly turning over compartment in the synthetic pathway between plasma FFA and VLDLTGFA, $\lambda_{3,1}$ is about half the value obtained with model
A. In models $A$ and $C$ the values for $\lambda_{0,5}$ are similar. However, in $B, \lambda_{0,5}$ is only about one-half the value found in $\mathrm{A}$ and $\mathrm{C}$. This difference arises from the fact that about one-half of the plasma VLDL-TGFA activity in $\mathrm{B}$ does not leave the plasma irreversibly but recycles through compartment 7 back to the plasma at a later time. Consequently, the sum of $\lambda_{0,5}$ and $\lambda_{7,5}$ in $\mathrm{B}$ (total fractional turnover rate of VLDL-TGFA) is similar to $\lambda_{0,5}$ alone in models $\mathrm{A}$ and $\mathrm{C}$. The rate constants for the slowly turning over compartments required to produce the late portion of the VLDL-TGFA activity curve (significant radioactivity still present at 1440 
min) are the smallest in magnitude $\left(\lambda_{5,8}\right.$ in $A, \lambda_{5,7}$ in $B$, and $\lambda_{1,8}$ in $C$ ).

These differences in parameter values from one model to another are accompanied by differences in the steadystate solutions. In models $B$ and $C, \rho_{8,1}$ (rate of hepatic uptake of plasma FFA destined for secretion as VLDLTGFA) is about one-half the value of $\rho_{3,1}$ calculated with model A. Estimation of the steady-state production rate (or rate of irreversible loss) of VLDL-TGFA $\left(\rho_{0,5}\right)$ is quite sensitive to the model chosen to fit the data. Models $A$ and $C$ both yield a value of $\rho_{0,5}$ between 70 and $80 \mu \mathrm{Eq} / \mathrm{min}$, while model $\mathrm{B}$ yields a value of about $43 \mu \mathrm{Eq} / \mathrm{min}$. In $\mathrm{A}$ and $\mathrm{B}$ all of $\rho_{0,5}$ is derived from plasma FFA. In $\mathrm{C}$, however, only a little more than one-half of $\rho_{0,5}$ is derived from plasma FFA; the remainder is derived from $U_{i n}$, an additional (unlabeled) source of fatty acid.

Certain steady-state measures remain relatively constant from one model to another. ${ }^{2}$ Just as the fractional rate of loss of plasma VLDL-TGFA (irreversible plus reversible) remains relatively invariant, so also does the total transport $\left(\rho_{5,5}\right)$ of VLDL-TGFA through the plasma compartment. $\rho_{5,4}$ (transport of plasma FFA to plasma VLDL-TGFA through the fast hepatic pathway) also remains invariant among the three models. These latter two relations imply that the magnitudes of the rate of delivery of VLDL-TGFA into the plasma from compartment $6\left(\rho_{5,6}\right)$ in A, from compartment 7 $\left(\rho_{0,7}\right)$ in $\mathrm{B}$, and from hepatic de novo synthesis or utilization of stored, preformed fatty acid $\left(\mathrm{U}_{\mathrm{B}}\right)$ in $\mathrm{C}$ must be similar. These rates were $38.4,35.0$, and $30.8 \mu \mathrm{Eq} /$ min, respectively. Estimates of $\rho_{0,5}$ for all three models can be summarized as follows: (a) model A, $\rho_{0,5}=\rho_{5,4}$ $+\rho_{5,8} ;(b)$ model $\mathrm{B}, \rho_{0,5}=\rho_{5,4} ;(c)$ model $\mathrm{C}, \rho_{0,5}=$ $\rho_{5,4}+U_{5}$. These relations demonstrate that interconver-

${ }^{2}$ It may be shown, using the integral equation approach, that some of the steady-state solutions, and hence their changes, are model independent. We chose not to use this method because of our desire to show all possible changes that may be brought about by changes in parameter values. sion of the steady-state solutions of the three models, especially with respect to $\rho_{0,5}$, is relatively easy to accomplish. ${ }^{3}$

\section{Model Testing}

A. Effects of $\lambda_{1,8}$ and $\lambda_{s, 4}$ on various measures of model $A$. As noted in the companion paper (10), the reliability of FFA radioactivity measurements in plasma beyond $90 \mathrm{~min}$ was limited because of very low levels of radioactivity and the possibility of significant contamination. Because of the inability to reliably determine the slope of the second exponential component of these data, a statistical constraint on the value of $\lambda_{1,2}$ was imposed on the least squares fit of model $A$ to the data. The relative effect of this constraint on other parameters and measures of the model was tested by refitting model $\mathrm{A}$ to the same steady-state and VLDLTGFA tracer data assuming various fixed values for $\lambda_{1,2}$ ranging from 300 to $33 \%$ of the original constrained value of $0.0177 \mathrm{~min}^{-1}$. The results of this test showed that most of the parameters and steady-state measures of model A were not sensitive to changes in $\lambda_{1,2}$ over a relatively wide range of values. Those measures exhibiting the largest changes are shown in Table III. The least squares fits of model $A$ to the VLDL-TGFA tracer data were not appreciably different from the original fit. Average deviations per datum were about 1 and $3 \%$ for $\lambda_{1,2}$ fixed at $0.531 \mathrm{~min}^{-1}$ and $.0059 \mathrm{~min}^{-1}$, respectively. Thus, even if the slope of the second expo-

\footnotetext{
${ }^{3}$ Havel (7) recently reported that FFA SA in the portal vein is less than arterial in the dog. The SA of FFA delivered to liver was, on the average, $20 \%(0-40 \%)$ less than that of arterial FFA. In the present studies the calculation of $\rho_{0,5}$ does not depend on this factor. For models with FFA input restricted to compartment 1, $\mathrm{U}_{1}=\frac{1}{\int_{0}{ }^{\infty}(\mathrm{SA})_{\mathbf{x}}} \mathrm{dt}$ where $\mathrm{SA}_{\mathbf{x}}$ is the specific activity anywhere in the system. Where there is an additional input pathway such as $U_{5}$, it by definition includes all dilutional effects anywhere in the system for TGFA synthesis.
}

TABLE III

Changes in Model A Parameters Resulting from $\lambda_{1,2}$ Being Changed to $300 \%$ and $33 \%$ of Its Original Value

\begin{tabular}{lccc}
\hline $\begin{array}{c}\text { Parameter } \\
\text { or measure }\end{array}$ & $\begin{array}{c}\lambda_{1,2} \text { equal } \\
\text { to } 0.0177 \mathrm{~min}^{-1}\end{array}$ & $\begin{array}{c}\lambda_{1,2} \text { increased } \\
\text { to } 0.0531 \mathrm{~min}^{-1}\end{array}$ & $\begin{array}{c}\lambda_{1,2} \text { decreased } \\
\text { to } 0.0059 \mathrm{~min}^{-1}\end{array}$ \\
\hline$\lambda_{3,1}\left(\mathrm{~min}^{-1}\right)$ & 0.0391 & 0.0355 & 0.0408 \\
$\lambda_{0,5} \quad "$ & 0.0178 & 0.0162 & 0.0185 \\
$M_{2}(\mu E q)$ & 4250 & 1417 & 12,749 \\
$\rho_{3,1}\left(\mu E q\right.$ min $\left.^{-1}\right)$ & 79.6 & 72.4 & 83.2 \\
$\rho_{0,5} \quad "$ & 79.6 & 72.4 & 83.2 \\
$\rho_{5,4} \quad "$ & 41.2 & 37.6 & 43.7 \\
$\rho_{5,6}$ & 38.4 & 34.8 & 39.5 \\
\hline
\end{tabular}




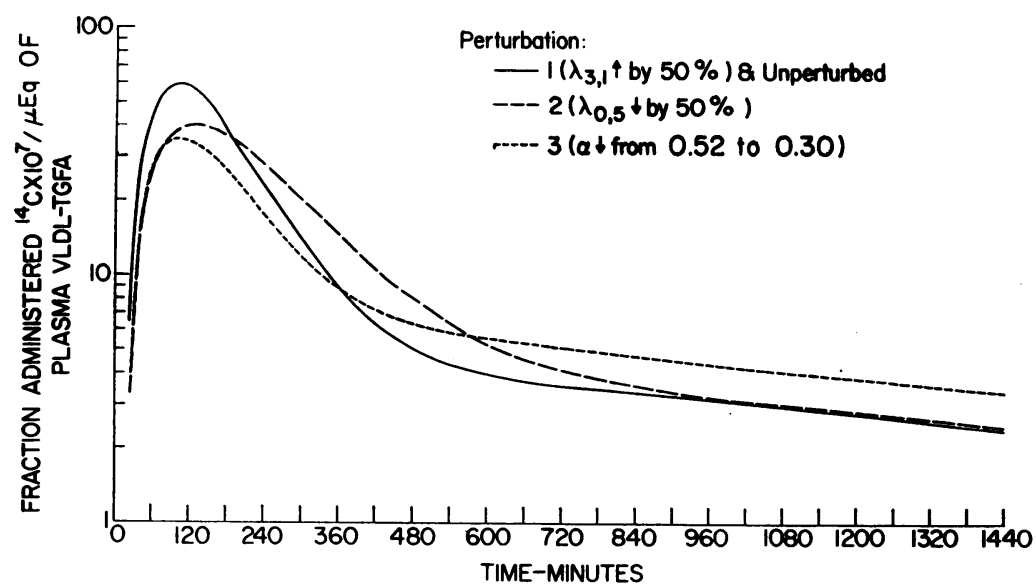

FIgure 3 'Simulated plasma VLDL-TGFA SA responses to palmitate- ${ }^{14} \mathrm{C}$ injection resulting from various perturbations in model A (Fig. 1 of companion paper). A $50 \%$ increase in $\lambda_{3,1}$ (solid line) shows no change from the original unperturbed state. Perturbation 2 represents a $50 \%$ decrease in $\lambda_{0,5}$. Perturbation 3 represents a decrease in the ratio $\rho_{5,4} / \rho_{5,5}(\alpha)$ from 0.52 to 0.30 .

nential component of the plasma FFA tracer data were grossly different from the value estimated, the calculated values for VLDL-TGFA production and removal from the plasma would not be markedly affected.

It was pointed out earlier that $\lambda_{5,4}$ was necessary in most studies to satisfy the shape of the experimental VLDL curve near its maximum. However, its value could not be determined with precision. A test of the sensitivity of other parameters to changes in the value of $\lambda_{5,4}$ showed considerable effects on $\lambda_{3,1}, \lambda_{0,5}$, and $\rho_{0,5}$ but negligible effect on all the others. Small changes in $\lambda_{5,4}$ could be almost completely offset by nearly equal (on a percentage basis) and opposite changes in $\lambda_{3,1}$. (Changes in $\lambda_{0,5}$ follow changes in $\lambda_{3,1}$ as a result of the continuity of mass-flow relation: $\lambda_{0,5} \mathrm{M}_{5}=\lambda_{3,1} \mathrm{M}_{1}$, where $\mathrm{M}_{1}$ and $\mathrm{M}_{5}$ are given.) The possible range of variation of $\lambda_{8,4}$ is quite large, nearly $50 \%$, and is greater for normal subjects than for patients. As a result the following considerations apply: $(a)$, the absolute precision of $\lambda_{3,1}$ and $\lambda_{0,5}$ (and $\rho_{0,5}$ ) due to potential variations in $\lambda_{5,4}$ is probably no greater than $50 \%$; $(b)$ the ratio $\lambda_{3,1} / \lambda_{0,5}$ is independent of variations in $\lambda_{5,4}$.

When considering perturbations due to changes in diet or comparing normal subjects with hyperlipoproteinemic patients, physiologic considerations may help in determining whether perturbations in $\lambda_{5,4}$ or in $\lambda_{3,1}$ are more likely. However, only if experimental data can be made more precise or direct measurements of other parameters in the model can be made will it be possible to reach an unequivocal decision on this score. The intercomparison of the three models shown in Table II is based on the constrained value of $\lambda_{5,4}$ as discussed above. Physiologic considerations support the hypothesis that perturbations influence FFA conversion to VLDL-
TGFA $\left(\lambda_{3,1}\right)$ and fractional removal of VLDL-TGFA $\left(\lambda_{0,5}\right)$ rather than the fractional release of VLDL from the liver $\left(\lambda_{5,4}\right)$, but there is no theoretical basis for choosing a specific value of $\lambda_{5,4}$ within the ranges indicated above.

$B$. Effects of parameter changes in model $A$ on parameters of models $B$ and $C$. In the companion paper (10) certain changes in the parameters of model A (mainly $\lambda_{3,1}, \lambda_{0,5}$ ) are reported in subjects on highcarbohydrate diets and in patients with type IV hyperlipoproteinemia. Corresponding changes (due to diet and disease) on the parameters of model $B$ and $C$ were also investigated. It was important in eliciting changes in parameter values to avoid effects due to the random fluctuations in the experimental data. (See Development of Compartmental Model-section C). Hence, using model $\mathrm{A}$ fitted to subject $1 \mathrm{~A}$ as a reference model, sets of VLDL-TGFA tracer and steady-state data were simulated to correspond to certain parameter changes in model $\mathrm{A}$, and the theoretical data thus derived were fitted to models $\mathrm{B}$ and $\mathrm{C}$ subject to the constraints stated above. (Development of Compartmental Modelsection C. Kinetic considerations: quantitative.)

The following parameter changes were introduced in model A: (1) $\lambda_{3,1}$ increased by $50 \%$ with a corresponding $50 \%$ increase in $\mathrm{M}_{5}$ (the sum $\lambda_{3,1}+\lambda_{0,1}$ was kept constant to keep the plasma FFA SA curve unchanged); (2) $\lambda_{0,5}$ was decreased $50 \%$ with a corresponding $100 \%$ increase in $\mathrm{M}_{5}$; (3) the ratio $\rho_{54} / \rho_{55}(\alpha)$ decreased from 0.52 to 0.30 with $M_{s}$ unchanged. The effects these changes would have on VLDL-TGFA SA curves are shown in Fig. 3.

Changes in the parameters and steady-state measures in models $\mathrm{B}$ and $\mathrm{C}$ due to perturbation (1) in model $\mathrm{A}$ 
TABLE IV

Changes in Models $A, B$, and $C$ Resulting from a $50 \%$ Increase in $\lambda_{3,1}$ of Model $A$

\begin{tabular}{|c|c|c|c|c|c|c|c|}
\hline & $\begin{array}{l}\text { Parameter } \\
\text { or measure }\end{array}$ & Model A & $\begin{array}{l}\text { Per cent } \\
\text { change }\end{array}$ & Model B & $\begin{array}{l}\text { Per cent } \\
\text { change }\end{array}$ & Model C & $\begin{array}{l}\text { Per cent } \\
\text { change }\end{array}$ \\
\hline$\lambda_{3,1}$ & $\left(\min ^{-1}\right)$ & 0.0587 & $\uparrow 50$ & 0.0314 & $\uparrow 50$ & 0.0303 & $\uparrow 50$ \\
\hline$\lambda_{0,1}$ & " & 0.204 & $\downarrow 9$ & 0.231 & $\downarrow<4$ & 0.120 & $\downarrow 8$ \\
\hline $\mathbf{M}_{1}$ & $(\mu E q)$ & 2040 & $\mathrm{NC}^{*}$ & 2040 & $\mathrm{NC}$ & 2040 & $\mathrm{NC}$ \\
\hline $\mathrm{M}_{5}$ & $"$ & 6720 & $\uparrow 50$ & 6720 & $\uparrow 50$ & 6720 & $\uparrow 50$ \\
\hline$M_{4}$ & $"$ & 4124 & $\uparrow 50$ & 4277 & $\uparrow 50$ & 4124 & $\uparrow 50$ \\
\hline$M_{6}$ & $"$ & 105,509 & $\uparrow 50$ & - & - & - & - \\
\hline$M_{7}$ & $"$ & - & - & 52,836 & $\uparrow 50$ & - & - \\
\hline $\mathrm{M}_{8}$ & $"$ & - & - & - & - & 255,890 & $\mathrm{NC}$ \\
\hline $\mathrm{U}_{1}$ & $\left(\mu E q \min ^{-1}\right)$ & 534 & $\mathrm{NC}$ & 534 & $\mathrm{NC}$ & 306 & $\mathrm{NC}$ \\
\hline $\mathrm{U}_{5}$ & $"$ & - & - & - & - & 46.2 & $\uparrow 50$ \\
\hline$\rho_{3,1}$ & $\left(\mu E q \min ^{-1}\right)$ & 119.4 & $\uparrow 50$ & 63.9 & $\uparrow 50$ & 61.8 & $\uparrow 50$ \\
\hline$\rho_{0,5}$ & $"$ & 119.4 & $\uparrow 50$ & 63.9 & $\uparrow 50$ & 108 & $\uparrow 50$ \\
\hline$\rho_{5,4}$ & $"$ & 61.8 & $\uparrow 50$ & 63.9 & $\uparrow 50$ & 61.8 & $\uparrow 50$ \\
\hline$\rho_{5,6}$ & $"$ & 57.6 & $\uparrow 50$ & - & - & - & - \\
\hline$\rho_{5,7}$ & $"$ & - & - & 52.5 & $\uparrow 50$ & - & - \\
\hline$\rho_{8,1}$ & $"$ & - & - & - & - & 263 & $\mathrm{NC}$ \\
\hline
\end{tabular}

* No change.

are shown in Table IV. The $50 \%$ increase in $\lambda_{81}$ of model $A$ results in a proportional increase in $M_{4}, M_{8}$, $M_{6}, \rho_{8,1}, \rho_{5,4}, \rho_{8,8}$, and $\rho_{0,5}$ but does not change the shape or magnitude of the VLDL-TGFA SA curve. The steady-state relations between models $B$ and $C$ and model A previously described (see Development of Compartmental Model-section D) are not altered. $\rho_{3,1}$, $\rho_{5,4}$ and $\rho_{0,5}$ in $\mathrm{B}$ and $\mathrm{C}$ are also increased by about $50 \%$. Furthermore, the $50 \%$ increase in $\rho_{5,6}$ of model $\mathrm{A}$ is paralleled in models $\mathrm{B}$ and $\mathrm{C}$ by a $50 \%$ increase in $\rho_{0,7}$ and $U_{5}$, respectively. Thus, a perturbation reflected in model $A$ as an increase in $M$ o resulting from an increase in $\lambda_{s, 1}$ would produce analogous and quantitatively parallel changes whichever model was used.

Changes in models $B$ and $C$ resulting from perturbation (2) in model A (a $50 \%$ decrease in $\lambda_{0,5}$ ) are shown in Table V. The decrease in $\lambda_{0,5}$ in model $A$ is reflected in $\mathrm{C}$ by a nearly proportional decrease in $\lambda_{0,5}$ and in $\mathrm{B}$ by a nearly proportional decrease in both $\lambda_{0,5}$ and $\lambda_{7,5}$. Consequently the ratio of $\left.\lambda_{0,5} / \lambda_{0,5}+\lambda_{7,8}\right)$ in $B$ is nearly

TABLE V

Changes in Models $A, B$, and C Resulting from a $50 \%$ Decrease in $\lambda_{0,5}$ of Model $A$

\begin{tabular}{|c|c|c|c|c|c|c|c|}
\hline & $\begin{array}{l}\text { Parameter } \\
\text { or measure }\end{array}$ & Model A & $\begin{array}{l}\text { Per cent } \\
\text { change }\end{array}$ & Model B & $\begin{array}{l}\text { Per cent } \\
\text { change }\end{array}$ & Model C & $\begin{array}{l}\text { Per cent } \\
\text { change }\end{array}$ \\
\hline$\lambda_{0,5}$ & & 0.00889 & $\downarrow 50$ & 0.00475 & $\downarrow 50$ & 0.00828 & $\downarrow 55$ \\
\hline$\lambda_{7,5}$ & $\left(\min ^{-1}\right)$ & - & - & 0.00366 & $\downarrow 53$ & - & - \\
\hline$\lambda_{5,5}$ & $"$ & 0.00889 & $\downarrow 50$ & 0.00841 & $\downarrow 51$ & 0.00828 & $\downarrow 55$ \\
\hline$M_{6}$ & $(\mu E q)$ & 8960 & $\uparrow 100$ & 8960 & $\uparrow 100$ & 8960 & $\uparrow 100$ \\
\hline $\mathrm{U}_{1}$ & $\left(\mu E q \min ^{-1}\right)$ & 534 & $\mathrm{NC}^{*}$ & 534 & $\mathrm{NC}$ & 300 & $\downarrow<2$ \\
\hline $\mathrm{U}_{5}$ & " & - & - & - & - & 31.5 & $\uparrow<2$ \\
\hline$\rho_{3,1}$ & $\left(\mu E q \min ^{-1}\right)$ & 79.6 & $\mathrm{NC}$ & 42.5 & $\downarrow<1$ & 42.7 & $\uparrow<4$ \\
\hline$\rho_{0,5}$ & $"$ & 79.6 & $\mathrm{NC}$ & 42.5 & $\downarrow<1$ & 74.2 & $\uparrow<3$ \\
\hline$\rho_{5,4}$ & $"$ & 41.2 & NC & 42.5 & $\downarrow<1$ & 42.7 & $\uparrow<4$ \\
\hline$\rho_{5,6}$ & $"$ & 38.4 & $\mathrm{NC}$ & - & - & - & - \\
\hline$\rho_{5,7}$ & $"$ & 一 & - & 32.8 & $\downarrow 6$ & - & - \\
\hline$\rho_{8,1}$ & $"$ & - & - & - & - & 267 & $<1$ \\
\hline
\end{tabular}

* No change. 
TABLE VI

Changes in Models $A, B$, and C Resulting from a Decrease in a of Model A from 0.52 to 0.30

\begin{tabular}{|c|c|c|c|c|c|c|c|}
\hline & $\begin{array}{l}\text { Parameter } \\
\text { or measure }\end{array}$ & Model A & $\begin{array}{l}\text { Per cent } \\
\text { change }\end{array}$ & Model B & $\begin{array}{l}\text { Per cent } \\
\text { change }\end{array}$ & Model C & $\begin{array}{l}\text { Per cent } \\
\text { change }\end{array}$ \\
\hline$\alpha$ & & 0.30 & $\downarrow 42$ & 1.0 & $\mathrm{NC}^{*}$ & 1.0 & $\mathrm{NC}$ \\
\hline$\lambda_{3,1}$ & $\left(\min ^{-1}\right)$ & 0.0391 & $\mathrm{NC}$ & 0.0127 & $\downarrow 40$ & 0.0125 & $\downarrow 38$ \\
\hline$\lambda_{0,1}$ & $"$ & 0.223 & $\mathrm{NC}$ & 0.249 & $\uparrow<3$ & 0.0829 & $\downarrow 36$ \\
\hline$\lambda_{5,6}$ & " & 0.00546 & $\mathrm{NC}$ & - & - & - & - \\
\hline$\lambda_{5,7}$ & $"$ & - & - & 0.00168 & $\uparrow 65$ & - & - \\
\hline$\lambda_{1,8}$ & $"$ & - & - & - & - & 0.00167 & $\uparrow 62$ \\
\hline$\lambda_{0,5}$ & " & 0.0178 & $\mathrm{NC}$ & 0.00578 & $\downarrow 40$ & 0.0156 & $\downarrow<3$ \\
\hline$\lambda_{7,5}$ & " & - & - & 0.0109 & $\uparrow 40$ & - & - \\
\hline$\lambda_{8,1}$ & $"$ & - & - & - & - & 0.196 & $\uparrow 52$ \\
\hline$M I_{1}$ & $(\mu E q)$ & 2040 & $\mathrm{NC}$ & 2040 & $\mathrm{NC}$ & 2040 & $\mathrm{NC}$ \\
\hline $\mathrm{M}_{5}$ & $"$ & 4480 & $\mathrm{NC}$ & 4480 & $\mathrm{NC}$ & 4480 & $\mathrm{NC}$ \\
\hline$M_{4}$ & $"$ & 1591 & $\downarrow 42$ & 1729 & $\downarrow 40$ & 1697 & $\downarrow 38$ \\
\hline $\mathrm{M}_{6}$ & $"$ & 102,180 & $\uparrow 45$ & - & - & - & - \\
\hline $\mathrm{M}_{7}$ & $"$ & - & - & 28,995 & $\downarrow 18$ & - & - \\
\hline $\mathrm{M}_{8}$ & $"$ & - & - & - & - & - & - \\
\hline $\mathrm{U}_{1}$ & $\left(\mu E q \min ^{-1}\right)$ & 534 & $\mathrm{NC}$ & 534 & $\mathrm{NC}$ & 195 & $\downarrow 36$ \\
\hline $\mathrm{U}_{5}$ & & - & - & - & - & 44.6 & $\uparrow 45$ \\
\hline$\rho_{3,1}$ & $\left(\mu E q \min ^{-1}\right)$ & 79.6 & $\mathrm{NC}$ & 25.9 & $\downarrow 40$ & 25.5 & $\downarrow 38$ \\
\hline$\rho_{0,5}$ & $"$ & 79.6 & $\mathrm{NC}$ & 25.9 & $\downarrow 40$ & 70.1 & $\downarrow<3$ \\
\hline$\rho_{5,4}$ & $"$. & 23.9 & $\downarrow 42$ & 25.9 & $\downarrow 40$ & 25.5 & $\downarrow 38$ \\
\hline$\rho_{5,6}$ & $"$ & 55.8 & $\uparrow 45$ & - & - & - & - \\
\hline$\rho_{5,7}$ & $"$ & - & - & 48.7 & $\uparrow 40$ & - & - \\
\hline$\rho_{8,1}$ & $"$ & - & - & - & - & 40.1 & $\uparrow 43$ \\
\hline$\rho_{5,5}$ & $"$ & 79.6 & $\mathrm{NC}$ & 74.6 & $\downarrow<1$ & 70.1 & $\downarrow<3$ \\
\hline \multicolumn{2}{|c|}{$\rho_{5,4} / \rho_{5,5} \ddagger$} & 0.30 & $\downarrow 42$ & 0.35 & $\downarrow 36$ & 0.36 & $\downarrow 37$ \\
\hline
\end{tabular}

* No change.

$\ddagger \rho_{5,4} / \rho_{5,5}$ equals $\alpha$ in model $\mathrm{A}$.

identical with that shown in Table II. Except for a doubling of $M_{5}$, the steady-state solutions of all three models show very little variation as a result of this perturbation.

The simulated plasma VLDL-TGFA SA data (Fig. 3) resulting from perturbation (2) in model A exhibit a lower maximum SA value at the peak of curve ( $\left.t_{\max }\right)$. Although total radioactivity in plasma VLDL-TGFA at $t_{\max }$ is increased, the $\mathrm{SA}$ is less because of a relatively greater increase in plasma VLDL-TGFA mass. The simulated data also show a decrease in the magnitude of the descending slope immediately following $t_{\max }$. It is worth noting that the value of $\lambda_{0,5}$ and the magnitude of this descending slope are positively correlated but not equal. While perturbation (2) represents a decrease in $\lambda_{0,5}$ from $0.0178 \mathrm{~min}^{-1}$ to $0.00889 \mathrm{~min}^{-1}$, the descending slope following $t_{\max }$ decreases from $0.0081 \mathrm{~min}^{-1}$ only to $0.0054 \mathrm{~min}^{-1}$. In all of the studies presented in the companion report (10) values of $\lambda_{0,5}$ in models $A$ and $C$ and $\lambda_{5,5}$ in model $B$ were considerably greater than the magnitude of the descending slope following $t_{\max }$. Thus, the slope of the $S A$ curve beyond $t_{\max }$, at which point the curve may appear to be first-order, would not yield a valid measure of the fractional rate of disappearance of $V L D L-T G F A$.

Perturbation (3) in model A (Table VI) represents a $42 \%$ decrease in the fraction of plasma FFA incorporated into plasma VLDL-TGFA via the fast pathway. Since total incorporation of plasma FFA into VLDLTGFA $\left(\rho_{3,1}\right)$ and transport of VLDL-TGFA through the plasma compartment $\left(\rho_{0,5}\right)$ are unchanged, the decrease in $\rho_{5,4}$ must be associated with a compensating increase in $\rho_{5,8}$. As a result of these changes, there was an increase in $\mathrm{M}_{6}$ and a decrease in $\mathrm{M}_{4}$.

This redistribution of plasma FFA transport to VLDLTGFA between the fast and slow pathways of model A is again paralleled by similar changes in models $B$ and C (Table VI). The increase in $\rho_{5,8}$ of $\mathrm{A}$ is paralleled by nearly proportional increases in $\rho_{5,7}$ and $U_{5}$ of $B$ and $C$, respectively. The decrease in $\rho_{5,4}$ of $A$ is associated with a nearly proportional decrease in $\rho_{3,1}, \rho_{5,4}$ and $\rho_{0,8}$ of B and $\rho_{3,1}$ and $\rho_{5,4}$ of C. As in the case of model A, $\rho_{0,8}$ of 
model $\mathrm{C}$ is essentially unchanged by this perturbation, but $\rho_{0,0}$ in model $\mathrm{B}$ is altered.

As seen in Fig. 3 a relative increase in the amount of FFA activity incorporated into plasma VLDL-TGFA through the slow hepatic pathway at the expense of the fast pathway has the expected effect on the shape of the VLDL-TGFA SA curve. The SA at later times, where the curve is flat, is increased, while the area under the earlier portion as well as the SA value at $t_{\max }$ are both decreased.

It can be concluded from these simulated perturbations on model $\mathrm{A}$ that variations in $\lambda_{3,1}, \lambda_{0,5}$ and their associated steady-state relations $\left(\rho_{3,1}\right.$ and $\left.\rho_{0,5}\right)$ are reflected in models $\mathrm{B}$ and $\mathrm{C}$ by corresponding and proportional changes. The only exception to this correspondence of parameter changes from model $\mathrm{A}$ to models $\mathrm{B}$ and $\mathrm{C}$ occurs in the case of an increase in $\rho_{0,5}$ secondary to an increase in $\rho_{5,8}$. This perturbation will not show up in model $\mathrm{B}$ as an increase in $\rho_{0,5}$ but as an increase in $\rho_{5,7}$ instead. Model $\mathrm{C}$, however, will reflect increases in $\rho_{0,5}$ and $U_{5}$ quantitatively similar to the respective increases in $\rho_{0,5}$ and $\rho_{5,8}$ of model A.

Physiological implications of models A, B, and C

All three models presented are equally consistent with the experimental data (tracer and tracee). However, differences in position of the slow compartment of each model lead to quite different physiological implications.

Model $\mathrm{C}$ suggests that the plasna FFA activity response to an injection of $\mathrm{FFA}-{ }^{14} \mathrm{C}$ can be described by a triexponential equation in which the slowest exponential, becoming dominant sometime after $4 \mathrm{hr}$, has a half-time greater than $10 \mathrm{hr}$ (Table II). This triexponential response is qualitatively consistent with the FFA tracer data obtained in man by Fredrickson and Gordon (16). Model $\mathrm{C}$, however, requires that a significant portion of plasma VLDL-TGFA $(>40 \%)$ be derived from fatty acid sources other than plasma FFA, i.e., $U_{s}$. This seems unlikely in view of the in vivo human studies performed by Havel (7) and Fine et al. (18). Havel demonstrated in about $75 \%$ of his normal subjects that the arterial VLDL-TGFA SA approached that of hepatic vein FFA within $4 \mathrm{hr}$ after the start of a constant infusion of palmitate $-{ }^{14} \mathrm{C}$, suggesting that the fraction of plasma VLDL-TGFA deriving from any source other than plasma FFA is negligible. Data obtained by Fine et al. (18) and further analyzed by us, using the integral equation approach employed by Havel (12), suggest that the fraction of plasma TGFA carbon deriving ultimately from glucose carbon is certainly less than $10 \%$ and probably less than $5 \%$ in both normal and hypertriglyceridemic patients on high carbohydrate diets undergoing sequential oral glucose loading.
Model A suggests the existence of a slowly turning over intermediate hepatic pool (compartment 6) between plasma FFA and VLDL-TGFA. There is some experimental justification for this. Havel et al. (19), Baker and Schotz (11), and Gross, Eigenbrodt, and Farquhar (20) have shown in the rabbit, rat, and dog, respectively, that a fraction of the hepatic TGFA turns over more slowly than plasma VLDL-TGFA. The data of Havel et al. (19) coupled with the electron microscopic studies of Jones, Ruderman, and Herrera (21) suggest that some of the plasma FFA extracted by liver accumulates as slowly turning over glyceride in cytoplasmic lipid droplets. If the fatty acid in these lipid droplets is eventually released to be incorporated into VLDLTGFA, model A might be a reasonable description of the normal physiology.

There are, however, several aspects of model A which are less than satisfactory. In some studies the calculated fatty acid mass of compartment $6\left(\mathrm{M}_{6}\right)$ is quite large. For normal and hypertriglyceridemic patients on a high carbohydrate diet (10), $\mathrm{M}_{\mathrm{B}}$ can exceed 75 g. Assuming a hepatic weight of $2 \%$ of body weight (22) and a TGFA content of about $1 \%$ of the wet weight of the liver $(1,2,23)$, the hepatic TGFA mass would be about $14 \mathrm{~g}$. If it is also assumed that the hepatic phospholipid content is about $2.5 \%$ (24) of wet weight of which about two-thirds is phospholipid fatty acid (PLFA), the hepatic PLFA mass would be about $23 \mathrm{~g}$. Thus, the total hepatic esterified fatty acid pool through which plasma $\mathrm{FFA}-{ }^{14} \mathrm{C}$ might pass en route to plasma VLDL-TGFA would be less than $40 \mathrm{~g}$. Furthermore, in two patients studied by Lieber and Spritz (23) and in the single patient studied by Farquhar et al. (1) on highcarbohydrate diets, the hepatic TG content was not increased.

Model A and model $\mathrm{C}$ both imply that the plasma response to a single injection of labeled VLDL-TGFA would be described by a single exponential with a rate constant equal to $\lambda_{0,5}$. Although this is consistent with the human studies of Farquhar et al. (1) it is not consistent with the more recent human studies of Havel (7) and Eaton et al. (8) or the studies of Havel et al. (19) and Laurell (25) in the rabbit and rat, respectively.

A third problem with models $\mathrm{A}$ and $\mathrm{C}$ is the fact that they do not take into account any direct hepatic uptake of plasma VLDL-TGFA as suggested by Havel et al. (19) in the rabbit and Stein and Shapiro (26) in the rat.

\footnotetext{
${ }^{4}$ The calculated hepatic esterified fatty acid mass of the sum of the delay compartments and compartment 4 did not exceed $2 \mathrm{~g}$ in any of our normal or hypertriglyceridemic subjects (10). Consequently, the total mass of the hepatic esterified fatty acid pool through which the ${ }^{14} \mathrm{C}$ moves before being secreted is close to $M_{6}$.
} 
Some of the difficulties with models $\mathrm{A}$ and $\mathrm{C}$ are overcome by model $\mathrm{B}$. Here it is assumed that compartment 7 is a pool of esterified fatty acid equilibrating with plasma VLDL-TGFA. It is unlikely that compartment 7 could correspond only to pools of other plasma lipoproteins exchanging with VLDL-TGFA since the calculated pool size for $M_{7}$ is considerably larger than that of the total circulating triglyceride pool. However, compartment 7 could represent the hepatic esterified fatty acid pool or a combination of this pool with other plasma lipoproteins since $\mathrm{M}_{7}$ is less than $20 \mathrm{~g}$. Model $\mathrm{B}$ also has other attractive features. The predicted response of plasma VLDL-TGFA activity after a single injection of labeled VLDL-TGFA would follow a biexponential curve like that found experimentally by Eaton et al. (8) and Havel (personal communication). Furthermore the model is consistent with the suggestion that the liver extracts (and recycles) VLDL-TGFA from plasma. As mentioned earlier (Table II) model B also implies a significantly lower production rate of VLDL-TGFA $\left(\rho_{0,5}\right)$ than models $A$ or $C$, since a large portion of the plasma
VLDL-TGFA leaves plasma only to reappear at a later time.

Model B, however, also seems to show an inconsistency with experimental data. The slope of the slower exponential component derived from a simulated plasma response to a single injection of labeled VLDL-TGFA is 5-10 times smaller than that observed in man by Havel.5 The data of Havel, obtained over a $4 \mathrm{hr}$ period, are limited by the shortness of the experimental interval. Nevertheless, they would seem to suggest that the late portion of the plasma VLDL-TGFA activity curve resulting from the injection of palmitate ${ }^{14} \mathrm{C}$ cannot be completely explained by a pool of esterified fatty acid equilibrating with plasma VLDL-TGFA.

The inconsistency of each model with various experimental observations implies that no one of them by itself is a completely acceptable description of FFATGFA transport in man. A more likely description would incorporate all three and perhaps even other models into one combination model which would be quantitatively

\footnotetext{
${ }^{5}$ Havel, R. J. Personal communication.
}
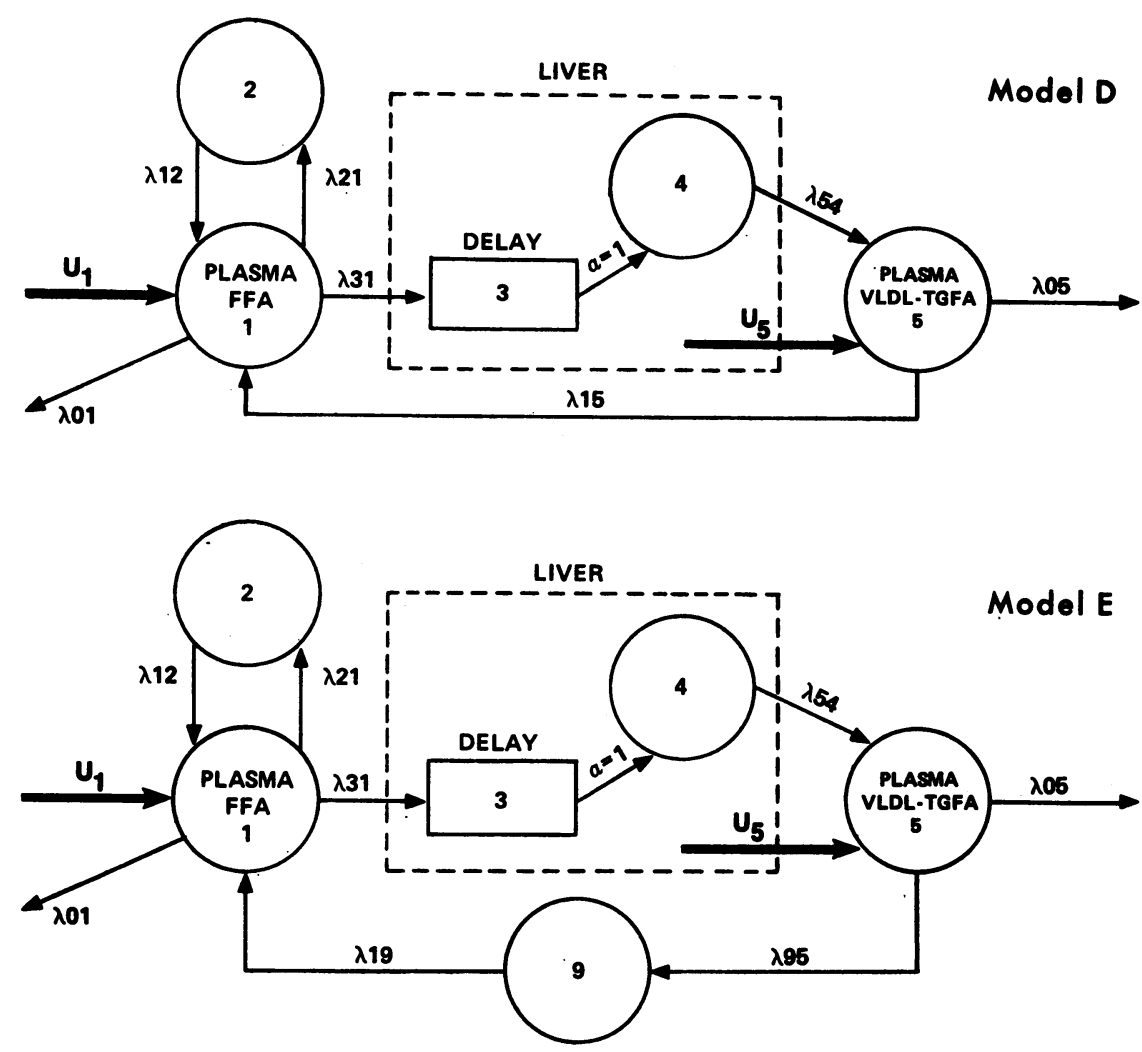

FIgURE 4 Two models describing recycling of plasma VLDL-TGFA through the plasma FFA pool. Both models are variants of model C (Fig. 1 of companion paper) without compartment 8 . In model $\mathrm{D}, \lambda_{0,5}$ and $\lambda_{1,5}$ equal $0.4 \lambda_{0,5}$ and $0.6 \lambda_{0,5}$, respectively, of model C. In model $\mathrm{E}, \lambda_{0,5}, \lambda_{8,5}$, and $\lambda_{1,8}$ equal $0.4 \lambda_{0,5}, 0.6 \lambda_{0,5}$, and $\lambda_{1,8}$, respectively, of model C. 

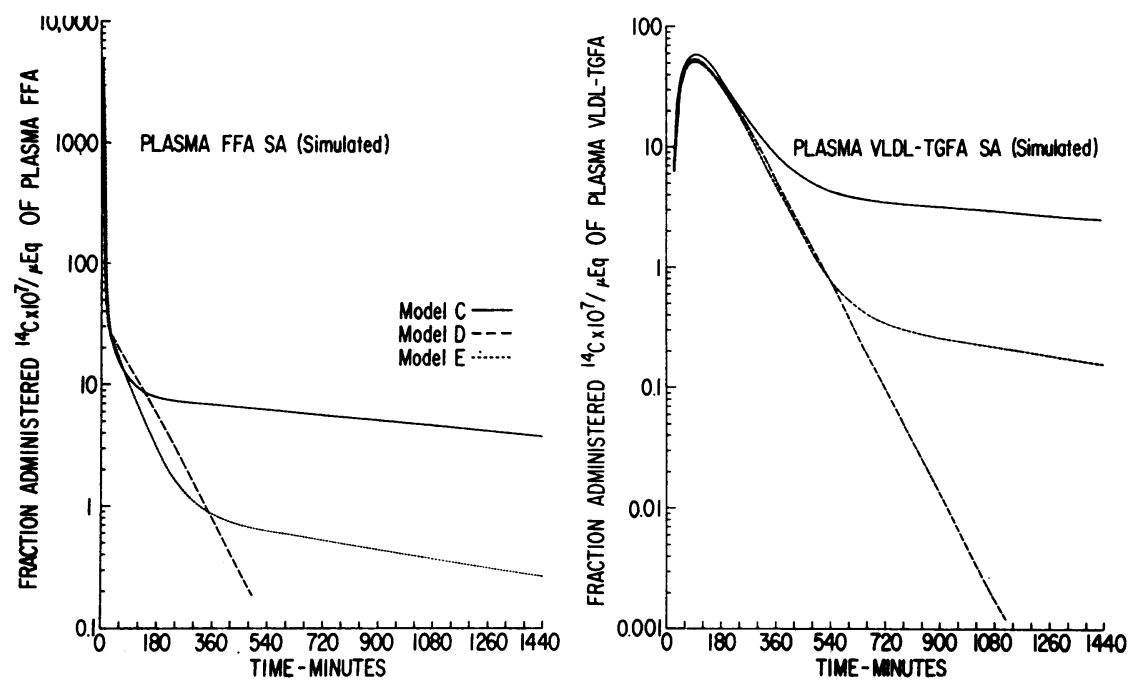

Figure 5 Simulated plasma FFA and VLDL-TGFA responses of models C, D, and $\mathrm{E}$ to a single injection of palmitate $-{ }^{14} \mathrm{C}$. Neither of the VLDL-TGFA responses of $\mathrm{D}$ or $\mathrm{E}$ is consistent with that of $\mathrm{C}$ which closely matches the experimental data of Fig. 2.

consistent with the pertinent data from the literature mentioned above as well as the data shown in Fig. 2. Unfortunately, available experimental data in man are inadequate to resolve uniquely the three slowly turning over compartments of such a combination model.

Other models of plasma FFA and VLDL-TGFA transport

A. Recycling of plasma VLDL-TGFA through the plasma FFA pool. It has been suggested by Eaton et al. (8) that about $60 \%$ of total plasma TGFA may be converted to plasma FFA through a relatively fast pathway. It could be hypothesized that this recycling of plasma TGFA through the plasma FFA pool could account for the late portion of the plasma VLDL-TGFA activity curve as opposed to compartments 6,7 , and 8 in models $\mathrm{A}, \mathrm{B}$, and $\mathrm{C}$, respectively. This hypothesis was tested using model D (Fig. 4), a variant of model $\mathrm{C}$ without compartment 8 . In model $\mathrm{D}, \lambda_{1,5}$ and $\lambda_{0,5}$ are set equal to $60 \%$ and $40 \%$, respectively, of the value of $\lambda_{0,5}$ in $\mathrm{C}$. All other parameters remain unchanged. Fig. 5 demonstrates the plasma FFA and VLDL-TGFA $\mathrm{SA}$ curves generated by model $\mathrm{D}$ superimposed on the respective SA responses of model $\mathrm{C}$. The late portion of the plasma FFA SA curve has too steep a slope to give rise to the relatively flat late portion of the VLDLTGFA curve.

Model E, also shown in Fig. 4 is a further modification of model $\mathrm{C}$ without compartment 8 . Here recycling of plasma VLDL-TGFA to plasma FFA takes place through a slowly turning over compartment 9 . In model $E, \lambda_{\theta, 5}$ and $\lambda_{1,8}$ are set equal to $\lambda_{1,5}$ in $D$ and $\lambda_{1,8}$ in $C$, respectively. This insures that the slope of the late portion of the FFA curve generated by model $\mathrm{E}$ will be similar to that generated by C. Fig. 5 reveals, however, that the magnitude of the late portion of the FFA $\mathrm{SA}$ curve generated by model $\mathrm{E}$ is less than $10 \%$ of that required to produce the proper magnitude of the late portion of the plasma VLDL-TGFA SA curve generated by model $\mathrm{C}$. Furthermore, data obtained by Eaton et al. (8) on the transport of total plasma TGFA to plasma FFA do not suggest movement through a slowly turning over pathway such as compartment 9 . Thus, the late portion of the plasma VLDL-TGFA SA data, occurring between 8 and $24 \mathrm{hr}$ following a pulse injection of $\mathrm{FFA}-{ }^{14} \mathrm{C}$, is not explained satisfactorily by recycling of plasma VLDL-TGFA through the plasma pool. However, significant recycling of plasma VLDLTGFA through the plasma FFA pool, if superimposed on models $\mathrm{A}, \mathrm{B}$, or $\mathrm{C}$, would be consistent with the data of Fig. 2 and is supported by the data of Eaton et al. (8).

B. Kinetic heterogeneity of the plasma VLDL-TGFA pool. There is little or no direct evidence in man suggesting kinetic heterogeneity within the plasma VLDLTGFA pool. However, such heterogeneity would be consistent with the biexponential plasma disappearance curve of injected labeled VLDL-TGFA (7). Consequently, VLDL-TGFA kinetic heterogeneity was investigated using models $\mathrm{F}$ and $\mathrm{G}$ (Fig. 6). Both models are variants of model $\mathrm{B}$ (Fig. 1 of the companion paper) without compartment 7 . Model $F$ assumes that the liver secretes two kinetically distinct species of VLDL-TGFA. One species (compartment 5) has a 

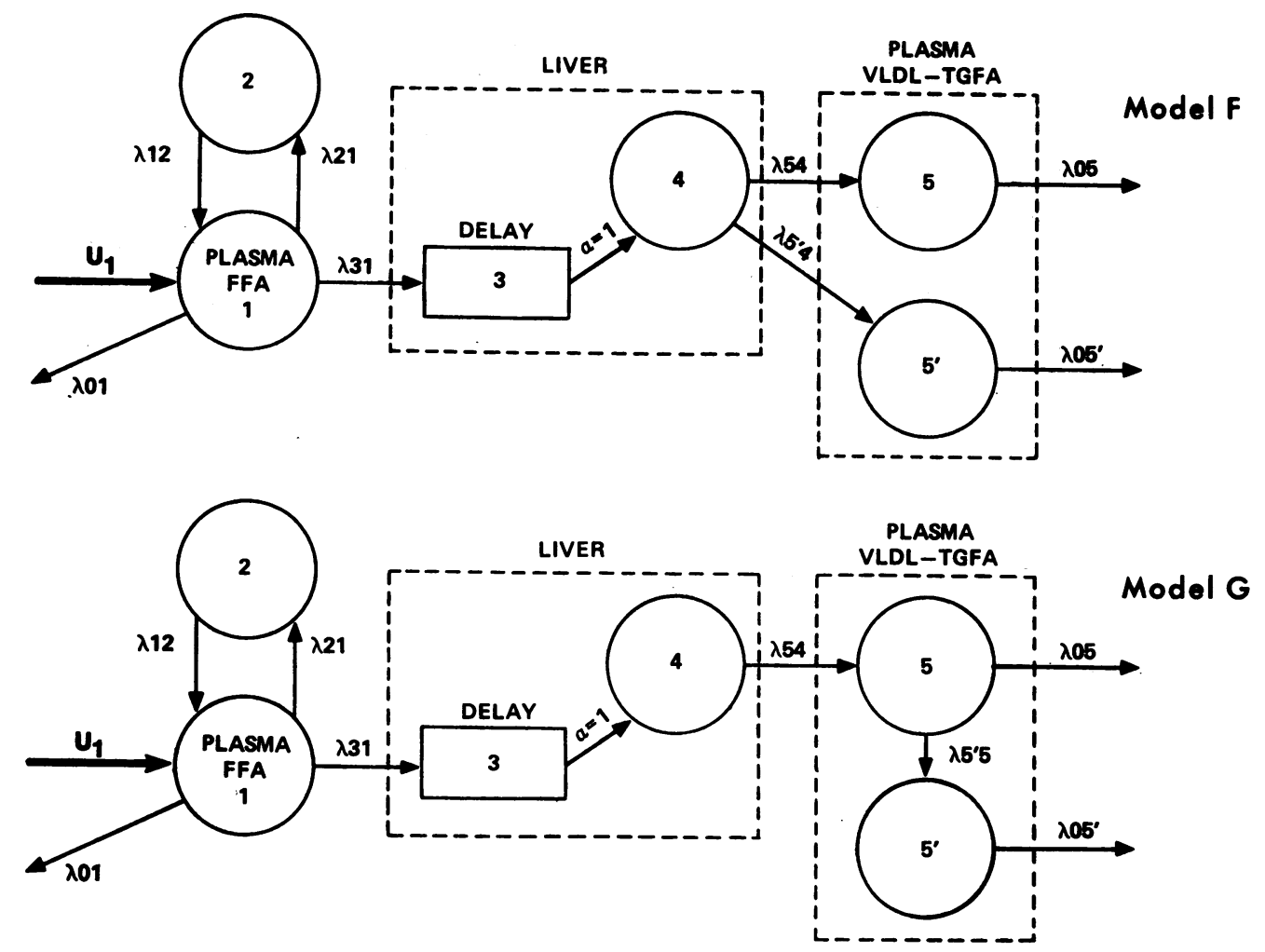

FIgUre 6 Two models describing kinetic heterogeneity of the plasma VLDL-TGFA pool. Model $\mathrm{F}$ assumes that the liver secretes two kinetically distinct species of VLDL-TGFA of widely different fractional turnover rates. Model $G$ assumes that the liver secretes a relatively rapidly turning over species of VLDL-TGFA which is partially converted in the plasma into a much more slowly turning over species of VLDL-TGFA.

fractional turnover rate $\left(\lambda_{0,5}\right)$ similar to $\lambda_{5,5}$ of model $\mathrm{B}$, while the other species (compartment $5^{\prime}$ ) has a much smaller fractional turnover rate $\left(\lambda_{0,5^{\prime}}\right)$ equal to about one-half the value of $\lambda_{5,7}$ of model B. Model G assumes that a relatively rapidly turning over species of VLDLTGFA (compartment 5 ) is secreted by the liver and partially converted in the plasma into a much more slowly turning over species of VLDL-TGFA (compartment $5^{\prime}$ ). Values of $\lambda_{0,5}$ and $\lambda_{0,5^{\prime}}$ of model $G$ are nearly equal to $\lambda_{0,5}$ and $\lambda_{0,5}$, of model $\mathrm{F}$.

Both models were consistent with the tracer and tracee data of Fig. 2. Steady-state solution of both reveals that the slowly turning over species of VLDLTGFA (responsible for the late portion of the VLDLTGFA (lata) accounted for about $50 \%$ of the total plasma VLDL-TGFA mass and $2.5 \%$ of the total plasma VLDL-TGFA transport. Although these two models (and variants of them) cannot be ruled out on the basis of the data of Fig. 2, their steady-state implications with respect to the ratios of $\mathrm{M}_{8} / \mathrm{M}_{8^{\prime}}$ (about 1) and $\rho_{0,5} / \rho_{0,5^{\prime}}$ (about 40 ) are not supported by any available evidence.
It might be hypothesized that the late portion of the plasma VLDL-TGFA tracer data (Fig. 2) represents contamination of VLDL-TGFA with the more slowly turning over LDL-TGFA. Such contamination could be described by model $G$ in which compartments 5 and $5^{\prime}$ represent VLDL-TGFA and LDL-TGFA, respectively. Although consistent with the tracer and tracee data of Fig. 2, the large size of $M_{5}$, implied by model $G$ argues against this hypothesis. Contamination of the late portion of the plasma VLDL-TGFA SA data by plasma FFA activity was negligible (10).

\section{Experiments Suggested by Models A, B, and C}

An experiment that would help determine the quantitative significance of compartment 7 in model $B$ and compartment 4 in models $\mathrm{A}, \mathrm{B}$, and $\mathrm{C}$ would be the injection of a labeled pulse of VLDL-TGFA. Observations of plasma VLDL-TGFA SA for 8-12 hr after such an injection would yield estimates of the size and minimum number of fatty acid pools exchanging with plasma VLDL-TGFA as well as help determine the relationship between compartments 4 and 5 in all three 
models. Such an experiment would also offer an upper limit estimate of the production rate of VLDL-TGFA. Monitoring the FFA SA over the same time interval would also help to determine the extent of plasma VLDL-TGFA recycling through the plasma FFA pool. Although similar experiments have been performed by Havel (7) and Eaton et al. (8), the results have been ambiguous due to the short duration of the experiments and the heterogeneity of lipoprotein labeling, respectively.

The availability of high SA VLDL-TGFA would also allow a more definitive study of the possible existence of VLDL-TGFA kinetic heterogeneity. 4-8 hr after a pulse injection of labeled VLDL-TGFA, the VLDLTGFA tracer could be aspirated from the plasma and reinjected. Significant kinetic heterogeneity of the plasma VLDL-TGFA pool would be considered unlikely if the plasma response to reinjected VLDL-TGFA tracer were similar to that resulting from the initial injection of labeled VLDL-TGFA. Important limitations of both of these reinjection experiments stem from the possible alterations of physical properties of VLDL resulting from the isolation techniques and the large quantities of radioactivity that would have to be injected into the donor subjects.

Another useful experiment to determine the necessity and significance of compartment 8 in model $\mathrm{C}$ would be the careful monitoring of plasma FFA SA for 8-12 hr after the injection of a pulse of $F F A-{ }^{14} \mathrm{C}$. The problem of low FFA SA would have to be circumvented by use of larger doses of radioactivity and very careful isolation procedures for plasma FFA.

Given an independent estimate of total liver weight, the determination of hepatic TGFA SA by liver biopsy could also be helpful in estimating the hepatic TGFA mass and activity at a particular time. Such data, suggesting an upper limit for the total exchangeable hepatic esterified fatty acid mass, would impose constraints on the maximum sizes calculated for compartments 6 and 7 in models $A$ and $B$, respectively.

Direct measurements of FFA and VLDL-TGFA activity gradients across the splanchnic bed for 8-12 $\mathrm{hr}$ after an injection or infusion of ${ }^{14} \mathrm{C}-\mathrm{FFA}$ would be helpful in determining the input-output response of the liver with respect to FFA and VLDL-TGFA. Such experiments have been performed by Carlson and Ekelund (27) and more recently by Havel (7) using hepatic vein catheterization. However, the short duration of these experiments, especially in hypertriglyceridemic patients, does not allow reliable estimation of the significance of compartments 6 and 7 in models $A$ and $B$, respectively.
Qualitative Comparison of Models A, B, and C With OTHER MODELS

The comparison of some of the parameters and steadystate measures of models $\mathrm{A}, \mathrm{B}$, and $\mathrm{C}$ with other models of FFA and TGFA transport requires some mention of the fundamental assumptions upon which other investigators have developed their models.

Nestel (2) obtained data similar to those shown in Fig. 2. He assumed that the fractional turnover rate $\left(\lambda_{\mathrm{TG}}\right)$ of plasma VLDL-TGFA was approximated by the descending slope of the plasma VLDL-TGFA activity curve following $t_{\max }$. This assumption can be questioned on theoretical as well as experimental grounds. As discussed above, $\lambda_{0,5}$ of models $\mathrm{A}$ and $\mathrm{C}$ can be more than twice the value of this slope and remain consistent with the tracer data of Fig. 2. Furthermore, recent data (7) on the plasma VLDL-TGFA response to the injection of labeled VLDL-TGFA suggest that $\lambda_{T a}$ is considerably greater than this slope. Since the production rate of VLDL-TGFA ( $\left.\rho_{\text {Tars }}\right)$ is given by the product of $\lambda_{T G}$ and the plasma VLDLTGFA mass ( $\mathrm{M}_{\mathrm{TGFA}}$ ), estimates of $\rho_{\mathrm{TGFA}}$ made by Nestel (2) could be spuriously low.

The objections to the estimation of $\lambda_{\text {Ta }}$ by the above technique are only partially overcome by the experiments of Farquhar et al. (1) who injected labeled glycerol instead of labeled FFA. In these studies the early descending slope of the glycerol-labeled plasma VLDL-TG activity curve is somewhat greater than that observed for the fatty acid-labeled VLDL-TGFA activity curve. Estimates of $\lambda_{\text {Ta }}$ by the initial slope technique using either tracer will come closer to our estimates based on models $\mathrm{A}, \mathrm{B}$, and $\mathrm{C}$ as the actual value of $\lambda_{\text {Ta }}$ increases. Havel (7) reported similar findings using his techniques.

The experiments and assumptions of Friedberg et al. (3) were similar to those of Nestel (2). However, plasma VLDL-TGFA were not separated from other lipoprotein TGFA. An integral equation approach was employed to determine the fraction of the injected FFA $-{ }^{14} \mathrm{C}$ which was converted to plasma TGFA. Combining this result with an independent estimate of the steady-state inflow of FFA into the plasma (UFA ), estimates were obtained for the rate of incorporation of plasma FFA into VLDL-TGFA ( the fraction of plasma VLDL-TGFA derived from plasma FFA. The quantitative validity of this analysis is difficult to assess. $\rho_{\text {FFA } \rightarrow \text { TGFA }}$ could be underestimated because of the assumption of a spuriously low value for

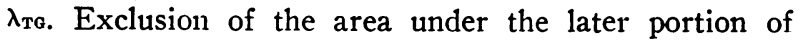
the plasma TGFA activity curve could also lead to underestimation of $\rho_{\text {FFA } \rightarrow \text { TaFA. }}$. Conversely, $\rho_{\text {FFA } \rightarrow \text { TOFA }}$ could be overestimated by use of the area under the total 
plasma TGFA activity curve instead of the smaller area under the plasma VLDL-TGFA activity curve. $\rho_{\text {FFA } \rightarrow \text { TarA }}$ could also be spuriously large because of failure to take account of the recycling of plasma FFA in the estimation of UFra.

Eaton et al. (8) have formulated a multicompartmental model fitted to plasma FFA and total TGFA SA data obtained over a $4 \mathrm{hr}$ interval after the injection of FFA $-{ }^{14} \mathrm{C}$. Because of the limited period of observation, these data contain little information about the slowly turning over compartments of models $\mathrm{A}, \mathrm{B}$, and C. Consequently the model formulated is similar to model $\mathrm{C}$ without compartment 8 . The absence of compartment 4 in Eaton's model results in estimates of $\lambda_{r G}$ somewhat less than those $\left(\lambda_{0,5}\right)$ obtained using model $\mathrm{C}$. In both models the differences between $\rho_{\text {FrA } \rightarrow \text { TGFA }}$ and $\rho_{\text {Tars }}$ are explained on the basis of a nonplasma source of fatty acid. Eaton et al. (8) pointed out that their published values of $\rho_{\text {TGFA }}$ could be high because VLDLTGFA were not separated from low density lipoprotein TGFA in the determination of plasma SA.

Ryan and Schwartz (4), Sailer et al. (5), and Miller et al. (6) have obtained data on the plasma FFA and total TGFA during a constant infusion of labeled FFA. They assume that the increase in activity of plasma TGFA from 60 to 120 min reflects the rate of entry of plasma FFA activity into plasma TGFA at the steady state. By combining this assumption with an estimate of the asymptotic value for plasma FFA SA, pFrA-TaFA is estimated. If it is further assumed that all plasma TGFA are derived from plasma FFA, $\rho_{\text {TGFA }}$ would be equal to $\rho_{F F A} \rightarrow$ TGFA. The fundamental assumption of this model is questionable. It can be shown by simulation using model $\mathrm{A}, \mathrm{B}$, or $\mathrm{C}$ that $\lambda_{\mathrm{T}}$ has a significant effect on dampening the rise of plasma TGFA activity during the interval from 60 to $120 \mathrm{~min}$. This effect is especially important in normal individuals whose $\lambda_{T a}$ is greater than that found in patients with hypertriglyceridemia (10). Havel (7) came to this same conclusion on experimental grounds. Consequently, the increase in TGFA activity over this time interval is a poor reflection of the steady-state rate of entry of plasma FFA activity into plasma TGFA. Furthermore, TGFA activity data over this time interval do not reflect movement of plasma FFA to TGFA through slowly turning over intermediate pools even though steady-state transport through such pathways could be quite large. As a result of these limitations, estimates of $\rho_{F F A} \rightarrow$ TGFA and p ${ }_{\text {TarA }}$ based on this model could be considerably lower than those suggested by models $\mathrm{A}, \mathrm{B}$, and $\mathrm{C} .^{\circ}$

\footnotetext{
- Estimates of $\rho_{\mathrm{FrA} \rightarrow \mathrm{TGFA}}$ made by Miller et al. (6) are considerably lower than those of Ryan and Schwartz (4) and Sailer et al. (5). This appears to result from a faulty assumption in the derivation of $\rho_{\mathrm{FFA} \rightarrow \mathrm{TGFA}}$. The expression
}

A valuable more direct approach has been taken recently by Havel (7) who measured gradients of radioactive FFA and VLDL-TGFA and net uptake of FFA across the splanchnic bed during a continuous infusion of $\mathrm{FFA}-{ }^{\mathrm{lt}} \mathrm{C}$ over a $4 \mathrm{hr}$ interval. Also obtaining the FFA mass gradient ${ }^{7}$ and total blood flow across the splanchnic bed, he formulated two models. The first (Havel model $\mathrm{I})^{8}$ utilizes the steady-state ratio of the activity gradients of VLDL-TGFA to FFA to obtain the fractional conversion (FC) of hepatic extracted FFA activity converted to VLDL-TGFA activity. $\rho_{F F A} \rightarrow$ TGFA is then obtained from the product of FC and the net steady-state splanchnic uptake of FFA. (Since it is assumed that all TGFA are derived from plasma FFA, $\rho_{\text {TGFA }}$ must equal $\rho_{\text {FFA } \rightarrow \text { TGFA. }}$ )

The estimates of $\rho_{\text {FFA } \rightarrow \text { TFGA }}$ based on Havel model I are open to some question. The magnitude of the splanchnic VLDL-TGFA activity gradient, being the small difference between two relatively large and similar numbers, becomes progressively more difficult to determine reliably after about $2 \mathrm{hr}$. Furthermore, if a significant fraction of the FFA activity extracted by the liver moves through a slowly turning over pool en route to VLDL-TGFA (e.g. model A), the value of FC obtained at $4 \mathrm{hr}$ would be smaller than the steady-state value

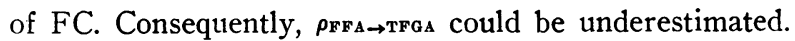
The possibility of underestimation is minimized, however, in those normal individuals whose arterial VLDLTGFA SA approached that of hepatic vein FFA at $4 \mathrm{hr}$. This implies not only that all plasma VLDLTGFA are derived from plasma FFA but also that FC has reached a maximum or near maximum value. However, FC could be underestimated in hypertriglyceridemic patients in whom the SA ratio of arterial VLDL-TGFA to hepatic vein FFA is less than 0.25 at $4 \mathrm{hr} .^{5}$ This possibility becomes especially pertinent in light of Havel's observation (7) that only about 55\% of the FFA activity being extracted by the liver is accounted for as $\mathrm{CO}_{2}$, ketone body, and VLDL-TGFA activity leaving the liver at $4 \mathrm{hr}$.

derived by Miller et al. (6) must be multiplied by the ratio of the plasma concentrations of TGFA to FFA before comparison with estimates of $\rho_{\text {FFA } \rightarrow \text { TGFA }}$ obtained by Ryan and Schwartz (4) and Sailer et al. (5) can be made.

${ }^{7}$ The mass concentration gradient of VLDL-TGFA across the splanchnic bed is too small to be resolved by the available experimental techniques (27).

${ }^{8}$ Additional assumptions are required by this model to obtain estimates of hepatic gradients from gradient data across the splanchnic bed. These are $(a)$ hepatic vein FFA SA approximates average FFA SA entering liver, (b) arterial FFA concentration approximates portal vein FFA concentration, and $(c)$ arterial VLDL-TGFA activity approximates portal vein VLDL-TGFA activity. These assumptions are suggested by experiments with dogs, in which the portal vein as well as hepatic vein and peripheral artery is monitored (30). 
The second model formulated by Havel (Havel model II) assumes that the arterial VLDL-TGFA activity response to a constant infusion of $\mathrm{FFA}-{ }^{14} \mathrm{C}$ over a $4 \mathrm{hr}$ period predominantly reflects the rate at which the activity of the plasma VLDL-TGFA pool approaches steady state. The arterial VLDL-TGFA SA data (after about $30 \mathrm{~min}$ ) are fitted by a single exponential component asymptotically approaching the SA of hepatic vein FFA. The magnitude of this exponential is assumed to equal $\lambda_{\text {ra. }}$ It can be readily shown that this model will underestimate $\lambda_{T G}$ and therefore $\rho_{T G E \Delta}$ if any intermediate or equilibrating hepatic pools (e.g. compartments 4 and 6 in model A or compartments 4 and 7 in model B) have not yet reached maximum SA by $30 \mathrm{~min}$. Estimates of $\rho_{\text {TGFA }}$ obtained from this model are most reliable (as in Havel model I) for those individuals whose SA ratio of arterial VLDL-TGFA to hepatic vein FFA approaches unity by $4 \mathrm{hr}$. However, even in these individuals the estimate of prars suggested by this model could be considerably less than that of Havel model I. Despite the above, the results obtained by Havel using Models I and II on normal subjects are similar. This agreement, however. may be fortuitous, and further studies are required.

\section{Quantitative Comparison of Models A, B, and C With OTher Models}

Quantitative differences among the various models can best be brought out by applying each of the models to the same set of data. Choosing $\rho_{\text {Tars }}$ as the measure for comparison, the data of the representative normal study shown in Fig. 2 were analyzed using the several models discussed above. The constant infusion response was simulated from the single injection data by convolution (28). The SA ratio of plasma VLDL-TGFA to plasma FFA with respect to these simulated data was about 0.5 at $4 \mathrm{hr}$. In applying Havel's models and those of Friedberg et al. (3) Ryan and Schwartz (4), the assumption was made that prors is equal to prFA $\rightarrow$ TGFA. It was also assumed in Havel's models that the FFA SA data of Fig. 2 approximated hepatic vein FFA SA.

The estimates of $\rho_{\text {TGFA }}$ obtained from models A, B, C, and the other models discussed are shown in Table VII. ${ }^{\circ}$ The largest estimates are obtained with models $A$ and $\mathrm{C}$ and that of Eaton et al. (8). The smaller value for model B has been discussed above (see Development of Compartmental Model). Except for Havel model II, the

\footnotetext{
' Estimates of $\rho_{\text {Tara }}$ by Havel models I and II and model $B$ would be about $20 \%$ greater than shown in Table VII, were the hepatic vein FFA SA to be $20 \%$ less than the antecubital vein FFA SA used in this analysis. $\rho_{\text {TGFA }}$ of models $\mathrm{A}$ and $\mathrm{C}$ would not change. However, $\rho_{5,4}$ in both of these models would be increased by about $20 \%$ at the expense of a concomitant decrease in $\rho_{5,6}$ and $U_{5}$ in models $A$ and $\mathrm{C}$, respectively.
}

TABLE VII

Calculated Values of $\rho$ TaFA Obtained from Several Models Fitted to Steady-State and Tracer Data of Study $1 \mathrm{~A}$

(Fig. 2)

\begin{tabular}{lc}
\hline \multicolumn{1}{c}{ Model } & $\rho_{\text {TGFA }}$ \\
\hline & $\left(\mu E q\right.$ min $\left.^{-1}\right)$ \\
A & 80 \\
B & 43 \\
C & 80 \\
Nestel & 37 \\
Friedberg et al. & 26 \\
Eaton et al. & 71 \\
Ryan and Schwartz & 15 \\
Havel I & 43 \\
Havel II & 22 \\
\hline
\end{tabular}

smallest values were obtained from the models of Friedberg et al. (3) and Ryan and Schwartz (4). The possible basis for the underestimation of $\rho_{\text {Tars }}$ by these two models as well as that of Nestel (2) has been discussed above.

It would appear from this quantitative comparison of various models that the best estimate of $\rho_{\text {Tars }}$ available at this time is a range of values. The minimal value of the range is given by Havel model I and model $\mathrm{B}$, while the maximum value is suggested by models $A$ and $C$ and perhaps by the model of Eaton et al. (8). All other models mentioned suggest values of $\rho_{\text {TGFA }}$ below the minimal value of this range. The estimate of $\rho_{\text {TarA }}$ made by Farquhar et al. (3) is difficult to compare because of the use of labeled glycerol rather than labeled FFA. However, it should be greater than $\rho_{\text {Tars }}$ estimated by Nestel (2) and probably would fall within the minimal and maximal values of the range defined above. Quantitative differences elucidated in these models relate to some extent to the particular set of data evaluated. If the SA ratio of arterial VLDL-TGFA to hepatic vein FFA were greater at $4 \mathrm{hr}$ than that implied by these data, differences in $\rho_{\text {TOFA }}$ among models $\mathrm{A}$ and $\mathrm{C}$ on one hand and model $\mathrm{B}$ and Havel model I on the other would have been less, and the range between minimal and maximal estimates of $\rho_{\text {TGFA }}$ would have been smaller. On the other hand these differences could have been greater were data from a hypertriglyceridemic patient used for the comparative analysis of $\rho_{\text {TOFA }}$.

The wide range of estimates of $\rho_{\text {TGFA }}\left(15-80 \mu \mathrm{Eq} \min ^{-1}\right)$ represents an example of how different kinetic models, all of which seem to be reasonable descriptions of the physiologic process ( $\left.\rho_{\text {TGFA }}\right)$, yield very different results even when applied to the same data. A number of explications for this disparity of estimates has been offered in terms of the more general logical framework of 
models $A, B$, and $C$, within which the fundamental assumptions of other models have been evaluated.

\section{ACKNOWLEDGMENT}

Partial support for this work was provided by Research Grant HE-12373, National Heart Institute, U. S. Public Health Service.

\section{REFERENCES}

1. Farquhar, J. W., R. C. Gross, R. M. Wagner, and G. M. Reaven. 1965. Validation of an incompletely coupled two-compartment nonrecycling catenary model for turnover of liver and plasma triglyceride in man. J. Lipid Res. 6: 119.

2. Nestel, P. J. 1965. Metabolism of linoleate and palmitate in patients with hypertriglyceridemia and heart disease. Metab. (Clin. Exp.) 14: 1 .

3. Friedberg, S. J., R. F. Klein, D. L. Trout, M. D. Bogdonoff, and E. H. Estes, Jr. 1961. The incorporation of plasma free fatty acids into plasma triglycerides in man J. Clin. Invest. 40: 1846.

4. Ryan, W. G., and T. B. Schwartz. 1965. Dynamics of plasma triglyceride turnover in man. Metab. (Clin. Exp.) 14: 1243 .

5. Sailer, S., F. Sandhofer, and H. Braunsteiner. 1966. Umsatzraten für freie Fettsäuren und Triglyceride im plasma bei essentieller Hyperlipämie. Klin. Wochenschr. 44: 1032

6. Miller, H. I., W. M. Bartz, and B. C. Durham. 1968 The rate of appearance of FFA in plasma triglyceride of normal and obese subjects. Metab. (Clin. Exp.) 17: 515.

7. Havel, R. J. 1968. Triglyceride and very low density lipoprotein turnover. In Proceedings of the 1968 Deuel Conference on Lipids. G. Cowgill, D. L. Estrich, and P. D. Wood, editors. Superintendent of Documents, U.S. Government Printing Office, Washington, D. C. 115.

8. Eaton, R. P., M. Berman, and D. Steinberg. 1969. Kinetic studies of plasma free fatty acid and triglyceride metabolism in man. J. Clin. Invest. 48: 1560.

9. Baker, N. 1969. The use of computers to study rates of lipid metabolism. J. Lipid Res. 10: 1.

10. Quarfordt, S., D. M. Shames, A. Frank, M. Berman, and D. Steinberg. 1970. Very low density lipoprotein triglyceride transport in type IV hyperlipoproteinemia and the effects of carbohydrate-rich diets. J. Clin. Invest. 49: 2281.

11. Baker, N., and M. C. Schotz. 1964. Use of multicompartmental models to measure rates of triglyceride metabolism in rats. J. Lipid Res. 5: 188.

12. Havel, R. J. 1961. Conversion of plasma free fatty acids into triglycerides of plasma lipoprotein fractions in man. Metab. (Clin. Exp.) 10: 1031.

13. Berman, M., and M. F. Weiss. 1962. The routine fitting of kinetic data to models: a mathematical formalism for digital computers. Biophys. J. 2: 275.
14. Berman, M., and M. F. Weiss. 1967. SAAM Manual. U. S. Public Health Service Publication No. 1703. U. S. Government Printing Office, Washington, D. C.

15. Berman, M. 1963. The formulation and testing of models. Ann. N. Y. Acad. Sci. 108: 182.

16. Fredrickson, D. S., and R. S. Gordon. 1958. The metabolism of albumin-bound ${ }^{14} \mathrm{C}$-labelled unesterified fatty acids in normal human subjects. J. Clin. Invest. 37: 1504.

17. Fredrickson, D. S., R. I. Levy, and R. S. Lees. 1967. Fat transport in lipoproteins-an integrated approach to mechanisms and disorders. N. Engl. J. Med. 276: 32.

18. Fine, M., G. Michaels, S. Shah, B. Chai, G. Fukayama, and L. Kinsell. 1962. The incorporation of ${ }^{14} \mathrm{C}$ from uniformly labelled glucose into plasma triglycerides in normals and hyperglyceridemics. Metab. (Clin. Exp.) 11: 893.

19. Havel, R. J., J. M. Felts, and C. M. Van Duyne. 1962. Formation and fate of endogenous triglycerides in blood plasma of rabbits. J. Lipid Res. 3: 297.

20. Gross, R. C., E. H. Eigenbrodt, and J. W. Farquhar. 1967. Endogenous triglyceride turnover in liver and plasma of the dog. J. Lipid Res. 8: 114

21. Jones, A. L., N. B. Ruderman, and M. G. Herrera. 1967. Electron microscopic and biochemical study of lipoprotein synthesis in the isolated perfused rat liver. J. Lipid Res. 8: 429.

22. Hargreaves, T. 1968. The Liver and Bile Metabolism. Appleton-Century-Crofts, New York. 4.

23. Lieber, C. S., and N. Spritz. 1966. Effects of prolonged ethanol intake in man: role of dietary, adipose and endogenously synthesized fatty acids in the pathogenesis of the alcoholic fatty liver. J. Clin. Invest. 45: 1400.

24. Martinsson, A., H. Sunzel, and B. Hood. 1963. Nitrogen, lipid, glycogen and deoxyribonucleic acid content of human liver. Acta Med. Scand. 173: 745.

25. Laurell, S. 1959. Recycling of intravenously injected palmitic acid $-1{ }^{14} \mathrm{C}$ as esterified fatty acid in the plasma of rats and turnover rate of plasma triglycerides. Acta Physiol. Scand. 47: 218.

26. Stein, Y., and B. Shapiro. 1960. Uptake and metabolism of triglycerides by the rat liver. J. Lipid Res. 1: 326.

27. Carlson, L. A., and L. Ekelund. 1963. Splanchnic production and uptake of endogenous triglycerides in the fasting state in man. J. Clin. Invest. 42: 714.

28. Stephenson, J. L. 1960. Integral equation description of transport phenomena in biological systems. In Proceedings of the Fourth Berkeley Symposium on Mathematical Statistics and Probability. University of California Press, Berkeley, Calif. 4: 335.

29. Brownell, G. L., M. Berman, and J. S. Robertson. 1968. Nomenclature for tracer kinetics. Int. J. Appl. Radiat. Isotop. 19: 249.

30. Basso, L. V., and R. J. Havel. 1970. Hepatic metabolism of free fatty acids in normal and diabetic dogs. J. Clin. Invest.. 49: 537. 